\title{
THE MODULI SPACE OF TWISTED CANONICAL DIVISORS
}

\author{
GAVRIL FARKAS ${ }^{1}$ AND RAHUL PANDHARIPANDE ${ }^{2}$ \\ ${ }^{1}$ Humboldt-Universität zu Berlin, Institut Für Mathematik, Unter den Linden 6, \\ 10099 Berlin, Germany (farkas@math.hu-berlin.de) \\ ${ }^{2}$ ETH Zürich, Department of Mathematics, Raemistrasse 101, 8092 Zürich, \\ Switzerland (rahul@math.ethz.ch)
}

(Received 8 October 2015; revised 4 March 2016; accepted 5 March 2016; first published online 5 April 2016)

\begin{abstract}
The moduli space of canonical divisors (with prescribed zeros and poles) on nonsingular curves is not compact since the curve may degenerate. We define a proper moduli space of twisted canonical divisors in $\overline{\mathcal{M}}_{g, n}$ which includes the space of canonical divisors as an open subset. The theory leads to geometric/combinatorial constraints on the closures of the moduli spaces of canonical divisors.

In case the differentials have at least one pole (the strictly meromorphic case), the moduli spaces of twisted canonical divisors on genus $g$ curves are of pure codimension $g$ in $\overline{\mathcal{M}}_{g, n}$. In addition to the closure of the canonical divisors on nonsingular curves, the moduli spaces have virtual components. In the Appendix A, a complete proposal relating the sum of the fundamental classes of all components (with intrinsic multiplicities) to a formula of Pixton is proposed. The result is a precise and explicit conjecture in the tautological ring for the weighted fundamental class of the moduli spaces of twisted canonical divisors.

As a consequence of the conjecture, the classes of the closures of the moduli spaces of canonical divisors on nonsingular curves are determined (both in the holomorphic and meromorphic cases).
\end{abstract}

Keywords: holomorphic differentials; moduli space of pointed curves; twisted canonical divisors

2010 Mathematics subject classification: $14 \mathrm{H} 10 ; 14 \mathrm{~N} 35 ; 14 \mathrm{~N} 10$

\section{Contents}

$\begin{array}{llr}0 & \text { Introduction } & \mathbf{6 1 6}\end{array}$

1 Twists of degenerating canonical bundles $\quad 622$

2 Dimension estimates $\quad \mathbf{6 3 0}$

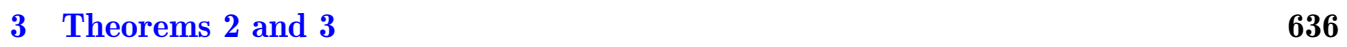

4 Sections of line bundles $\quad 638$

$5 \quad$ Twisted canonical divisors and limits of theta characteristics $\quad 643$

$6 \quad$ Twisted $k$-canonical divisors $\quad 655$

$\begin{array}{lr}\text { Acknowledgments } & 657\end{array}$ 
Appendix A. The weighted fundamental class of $\tilde{\mathcal{H}}_{g}(\mu)$ by F. Janda, R. Pandharipande, A. Pixton, and D. Zvonkine

\section{Introduction}

\subsection{Zeros and poles}

Let $\mathcal{M}_{g}$ be the moduli space of nonsingular curves of genus $g \geqslant 2$. The Hodge bundle,

$$
\mathbb{E} \rightarrow \mathcal{M}_{g}
$$

has fiber over the moduli point $[C] \in \mathcal{M}_{g}$ given by the space of holomorphic differentials $H^{0}\left(C, \omega_{C}\right)$. The projectivization

$$
\mathcal{H}_{g}=\mathbb{P}(\mathbb{E}) \rightarrow \mathcal{M}_{g}
$$

is a moduli space of canonical divisors on nonsingular curves. We may further stratify $\mathcal{H}_{g}$ by canonical divisors with multiplicities of zeros specified by a partition $\mu$ of $2 g-2$. Neither $\mathcal{H}_{g}$ nor the strata with specified zero multiplicities are compact. We describe a natural compactification of the strata associated to a partition $\mu$.

Meromorphic differentials arise naturally in the analysis of the boundary of the spaces of holomorphic differentials. We consider meromorphic differentials on curves with prescribed zero and pole multiplicities. Let

$$
\mu=\left(m_{1}, \ldots, m_{n}\right), \quad m_{i} \in \mathbb{Z}
$$

satisfy $\sum_{i=1}^{n} m_{i}=2 g-2$. The vector $\mu$ prescribes the zero multiplicities of a meromorphic differential via the positive parts $m_{i}>0$ and the pole multiplicities via the negative parts $m_{i}<0$. Parts with $m_{i}=0$ are also permitted (and correspond to points on the curve which are neither zeros nor poles and otherwise unconstrained).

Let $g$ and $n$ be in the stable range $2 g-2+n>0$. For a vector $\mu$ of length $n$, we define the closed substack $\mathcal{H}_{g}(\mu) \subset \mathcal{M}_{g, n}$ by

$$
\mathcal{H}_{g}(\mu)=\left\{\left[C, p_{1}, \ldots, p_{n}\right] \in \mathcal{M}_{g, n} \mid \mathcal{O}_{C}\left(\sum_{i=1}^{n} m_{i} p_{i}\right)=\omega_{C}\right\} .
$$

Consider first the case where all parts of $\mu$ are nonnegative. Since $\mathcal{H}_{g}(\mu)$ is the locus of points

$$
\left[C, p_{1}, \ldots, p_{n}\right] \in \mathcal{M}_{g, n}
$$

for which the evaluation map

$$
H^{0}\left(C, \omega_{C}\right) \rightarrow H^{0}\left(C, \omega_{\left.C \mid m_{1} p_{1}+\cdots+m_{n} p_{n}\right)}\right)
$$

is not injective, every component of $\mathcal{H}_{g}(\mu)$ has dimension at least $2 g-2+n$ in $\mathcal{M}_{g, n}$ by degeneracy loci considerations [13]. Polishchuk [25] has shown that $\mathcal{H}_{g}(\mu)$ is a nonsingular substack of $\mathcal{M}_{g, n}$ of pure dimension $2 g-2+n$. In fact, the arguments of [25] can be 
extended to the case where the vector $\mu$ has negative parts. ${ }^{1}$ In the strictly meromorphic case, $\mathcal{H}_{g}(\mu)$ is a nonsingular substack of dimension $2 g-3+n$ in $\mathcal{M}_{g, n}$.

In the holomorphic case, the spaces $\mathcal{H}_{g}(\mu)$ have been intensely studied from the point of view of flat surfaces (which leads to natural coordinates and a volume form); see [9]. However, an algebrogeometric study of the strata has been neglected: basic questions concerning the birational geometry and the cohomology classes of the strata of differentials are open.

We define here a proper moduli space of twisted canonical divisors

$$
\tilde{\mathcal{H}}_{g}(\mu) \subset \overline{\mathcal{M}}_{g, n}
$$

which contains $\mathcal{H}_{g}(\mu)$ as an open set

$$
\mathcal{H}_{g}(\mu) \subset \tilde{\mathcal{H}}_{g}(\mu) .
$$

The space $\widetilde{\mathcal{H}}_{g}(\mu)$ will typically be larger than the closure

$$
\overline{\mathcal{H}}_{g}(\mu) \subset \widetilde{\mathcal{H}}_{g}(\mu)
$$

We prove that every irreducible component of $\tilde{\mathcal{H}}_{g}(\mu)$ supported entirely in the boundary of $\overline{\mathcal{M}}_{g, n}$ has codimension $g$ in $\overline{\mathcal{M}}_{g, n}$.

In the strictly meromorphic case where there exists an $m_{i}<0$, the moduli space $\widetilde{\mathcal{H}}_{g}(\mu)$ is of pure codimension $g$ in $\overline{\mathcal{M}}_{g, n}$. The closure

$$
\overline{\mathcal{H}}_{g}(\mu) \subset \widetilde{\mathcal{H}}_{g}(\mu)
$$

is a union of irreducible components. The components of $\widetilde{\mathcal{H}}_{g}(\mu)$ which do not lie in $\overline{\mathcal{H}}_{g}(\mu)$ are called virtual. The virtual components play a basic role in the study of canonical divisors.

\subsection{Pixton's formula}

A relation to a beautiful formula of Pixton for an associated cycle class in $R^{g}\left(\overline{\mathcal{M}}_{g, n}\right)$ is explored in the Appendix A: the contributions of the virtual components of $\widetilde{\mathcal{H}}_{g}(\mu)$ are required to match Pixton's formula. The result is a precise and explicit conjecture in the tautological ring of $\overline{\mathcal{M}}_{g, n}$ for the sum of the fundamental classes (with intrinsic multiplicities) of all irreducible components of $\widetilde{\mathcal{H}}_{g}(\mu)$ in the strictly meromorphic case.

In the holomorphic case where all $m_{i} \geqslant 0$, the connection to Pixton's formula is more subtle since

$$
\widetilde{\mathcal{H}}_{g}(\mu) \subset \bar{M}_{g, n}
$$

is not of pure codimension $g$. The more refined approach to the moduli of canonical divisors proposed by Janda [19] via relative Gromov-Witten theory and virtual fundamental classes will likely be required to understand the holomorphic case (and to prove the conjecture of the Appendix A in the strictly meromorphic case). However, the virtual components clearly also play a role in the holomorphic case.

${ }^{1}$ Occurrences of $\omega_{C}$ in Polishchuk's argument should be replaced by $\omega_{C}\left(\sum_{i=1}^{\widehat{n}}\left|m_{i}\right| p_{i}\right)$ where $m_{1}, \ldots, m_{\widehat{n}}$ are the negative parts of $\mu$. The reason for the change from codimension $g-1$ in the holomorphic case to codimension $g$ in the strictly meromorphic case is that $\omega_{C}$ is special and $\omega_{C}\left(\sum_{i=1}^{\widehat{n}}\left|m_{i}\right| p_{i}\right)$ is not. 
Remarkably, the fundamental classes of the varieties of closures

$$
\overline{\mathcal{H}}_{g}(\mu) \subset \overline{\mathcal{M}}_{g, n},
$$

in both the holomorphic and strictly meromorphic cases, are determined in the Appendix A as a consequence of the conjectured link between Pixton's formula and the weighted fundamental class of the moduli space of twisted canonical divisors in the strictly meromorphic case.

\subsection{Twists}

Let $\left[C, p_{1}, \ldots, p_{n}\right]$ be a Deligne-Mumford stable $n$-pointed curve. ${ }^{2}$ Let

$$
\mathrm{N}(C) \subset C
$$

be the nodal locus. A node $q \in \mathrm{N}(C)$ is basic if $q$ lies in the intersection of two distinct irreducible components of $C$. Let

$$
\mathrm{BN}(C) \subset \mathrm{N}(C)
$$

be the set of basic nodes, and let

$$
\widetilde{\mathrm{BN}}(C) \rightarrow \mathrm{BN}(C)
$$

be the canonical double cover defined by

$$
\widetilde{\mathrm{BN}}(C)=\{(q, D) \mid q \in \mathrm{BN}(C), q \in D \text {, and } D \subset C \text { an irreducible component }\} .
$$

A twist $I$ of the curve $C$ is a function

$$
I: \widetilde{\mathrm{BN}}(C) \rightarrow \mathbb{Z}
$$

satisfying the balancing, vanishing, sign, and transitivity conditions defined as follows.

Balancing: If a basic node $q$ lies in the intersection of distinct irreducible components $D, D^{\prime} \subset C$, then

$$
I(q, D)+I\left(q, D^{\prime}\right)=0 .
$$

Let $\operatorname{Irr}(C)$ be the set of irreducible components of $C$. If

$$
D, D^{\prime} \in \operatorname{Irr}(C)
$$

and there exists $q \in D \cap D^{\prime}$ with $I(q, D)=0$, we write

$$
D \approx D^{\prime}
$$

By the balancing condition, $\approx$ is symmetric. Let $\sim$ be the minimal equivalence relation on $\operatorname{Irr}(C)$ generated by $\approx$.

Vanishing: If $D, D^{\prime} \in \operatorname{Irr}(C)$ are distinct irreducible components in the same $\sim$-equivalence class and $q \in D \cap D^{\prime}$, then

$$
I(q, D)=I\left(q, D^{\prime}\right)=0 .
$$

\footnotetext{
${ }^{2}$ The definition of a twist given here is valid for any connected nodal curve.
} 
Sign: Let $q \in D \cap D^{\prime}$ and $\widehat{q} \in \widehat{D} \cap \widehat{D}^{\prime}$ be basic nodes of $C$. If $D \sim \widehat{D}$ and $D^{\prime} \sim \widehat{D}^{\prime}$, then

$$
I(q, D)>0 \Longrightarrow I(\widehat{q}, \widehat{D})>0 .
$$

Let $\operatorname{Irr}(C)^{\sim}$ be the set of $\sim$-equivalence classes. We define a directed graph $\Gamma_{I}(C)$ with vertex set $\operatorname{Irr}(C)^{\sim}$ by the following construction. A directed edge

$$
v \rightarrow v^{\prime}
$$

is placed between equivalence classes $v, v^{\prime} \in \operatorname{Irr}(C)^{\sim}$ if there exist

$$
D \in v, \quad D^{\prime} \in v^{\prime} \quad \text { and } \quad q \in D \cap D^{\prime}
$$

satisfying $I(q, D)>0$.

The vanishing condition prohibits self-edges at the vertices of $\Gamma_{I}(C)$. By the sign condition, vertices are not connected by directed edges in both directions: there is at most a single directed edge between vertices. The fourth defining condition for a twist $I$ is easily stated in terms of the associated graph $\Gamma_{I}(C)$.

Transitivity: The graph $\Gamma_{I}(C)$ has no directed loops.

If $C$ is curve of compact type, distinct irreducible components of $C$ intersect in at most one point. The vanishing and sign conditions are therefore trivial. Since $\Gamma_{I}(C)$ is a tree, the transitivity condition is always satisfied. In the compact type case, only the balancing condition is required for the definition of a twist.

\subsection{Twisted canonical divisors}

Let $\left[C, p_{1}, \ldots, p_{n}\right] \in \overline{\mathcal{M}}_{g, n}$, and let

$$
I: \widetilde{\mathrm{BN}}(C) \rightarrow \mathbb{Z}
$$

be a twist. Let $\mathrm{N}_{I} \subset \mathrm{BN}(C)$ be the set of basic nodes at which $I$ is nonzero,

$$
\mathrm{N}_{I}=\{q \in \mathrm{BN}(C) \mid I(q, D) \neq 0 \text { for } q \in D\} .
$$

Associated to $I$ is the partial normalization

$$
v: C_{I} \rightarrow C
$$

defined by normalizing exactly the nodes in $\mathrm{N}_{I}$. The curve $C_{I}$ may be disconnected.

For a node $q \in \mathrm{N}_{I}$ in the intersection of distinct components $D^{\prime}$ and $D^{\prime \prime}$ of $C$, we have $v^{-1}(q)=\left\{q^{\prime}, q^{\prime \prime}\right\}$. Let

$$
D_{q}^{\prime} \subset v^{-1}\left(D^{\prime}\right) \quad \text { and } \quad D_{q}^{\prime \prime} \subset v^{-1}\left(D^{\prime \prime}\right)
$$

denote the irreducible components of $C_{I}$ such that $q^{\prime} \in D_{q}^{\prime}$ and $q^{\prime \prime} \in D_{q}^{\prime \prime}$. By the definition of $v$ and the sign condition,

$$
D_{q}^{\prime} \cap D_{q}^{\prime \prime}=\emptyset \quad \text { in } C_{I} .
$$

Let $\mu=\left(m_{1}, \ldots, m_{n}\right)$ be a vector satisfying $\sum_{i=1}^{n} m_{i}=2 g-2$. To the stable curve $\left[C, p_{1}, \ldots, p_{n}\right]$, we associate the Cartier divisor $\sum_{i=1}^{n} m_{i} p_{i}$ on $C$. 
Definition 1. The divisor $\sum_{i=1}^{n} m_{i} p_{i}$ associated to $\left[C, p_{1}, \ldots, p_{n}\right]$ is twisted canonical if there exists a twist $I$ for which

$$
v^{*} \mathcal{O}_{C}\left(\sum_{i=1}^{n} m_{i} p_{i}\right) \stackrel{\simeq}{=} v^{*}\left(\omega_{C}\right) \otimes \mathcal{O}_{C_{I}}\left(\sum_{q \in \mathrm{N}_{I}} I\left(q, D_{q}^{\prime}\right) \cdot q^{\prime}+I\left(q, D_{q}^{\prime \prime}\right) \cdot q^{\prime \prime}\right)
$$

on the partial normalization $C_{I}$.

We define the subset $\widetilde{\mathcal{H}}_{g}(\mu) \subset \overline{\mathcal{M}}_{g, n}$ parameterizing twisted canonical divisors by

$$
\widetilde{\mathcal{H}}_{g}(\mu)=\left\{\left[C, p_{1}, \ldots, p_{n}\right] \in \overline{\mathcal{M}}_{g, n} \mid \sum_{i=1}^{n} m_{i} p_{i} \text { is a twisted canonical divisor }\right\} .
$$

By definition, we have

$$
\widetilde{\mathcal{H}}_{g}(\mu) \cap \mathcal{M}_{g, n}=\mathcal{H}_{g}(\mu),
$$

so $\mathcal{H}_{g}(\mu) \subset \tilde{\mathcal{H}}_{g}(\mu)$ is an open set.

If $\mu$ has a part equal to 0 , we write $\mu=\left(\mu^{\prime}, 0\right)$. Let

$$
\tau: \overline{\mathcal{M}}_{g, n} \rightarrow \overline{\mathcal{M}}_{g, n-1}
$$

be the map forgetting the 0 part (when permitted by stability). As a straightforward consequence of Definition 1, we obtain

$$
\widetilde{\mathcal{H}}_{g}(\mu)=\tau^{-1}\left(\tilde{\mathcal{H}}_{g}\left(\mu^{\prime}\right)\right)
$$

Theorem 2. If all parts of $\mu$ are nonnegative, $\tilde{\mathcal{H}}_{g}(\mu) \subset \overline{\mathcal{M}}_{g, n}$ is a closed substack with irreducible components of dimension either $2 g-2+n$ or $2 g-3+n$. The substack

$$
\overline{\mathcal{H}}_{g}(\mu) \subset \tilde{\mathcal{H}}_{g}(\mu)
$$

is the union of the components of dimension $2 g-2+n$.

Theorem 3. If $\mu$ has a negative part, $\widetilde{\mathcal{H}}_{g}(\mu) \subset \overline{\mathcal{M}}_{g, n}$ is a closed substack of pure dimension $2 g-3+n$ which contains

$$
\overline{\mathcal{H}}_{g}(\mu) \subset \tilde{\mathcal{H}}_{g}(\mu)
$$

as a union of components.

Theorems 2 and 3 constrain the closures of the strata of holomorphic and meromorphic differentials in geometric and combinatorial terms depending only on the partial normalization $C_{I}$ of $C$. By degree considerations on the curve $\left[C, p_{1}, \ldots, p_{n}\right]$, there are only finitely many twists $I$ which are relevant to determining whether $\sum_{i=1}^{n} m_{i} p_{i}$ is a twisted canonical divisor. ${ }^{3}$

Because of the virtual components in the boundary, Theorems 2 and 3 do not characterize the closure $\overline{\mathcal{H}}_{g}(\mu)$. Further constraints can easily be found on the closure

${ }^{3} \mathrm{~A}$ proof is given in Lemma 10 of $\S 2.3$ below. 
via residue conditions. Our perspective is not to exclude the virtual components, but rather to include them. In the strictly meromorphic case, the virtual components in the boundary are described in Lemma 13 via star graphs defined in $\S 2.2$. The virtual components may be seen as a shadow of the virtual fundamental class of an approach to the moduli space of differentials via relative stable maps proposed by Janda [19].

In $\S 5$, we discuss the relationship between twisted canonical divisors and spin curves via theta characteristics in case all the parts of $\mu$ are positive and even. The basic criterion of Proposition 16 of $\S 4.2$ for the smoothability of a twisted canonical divisor in the holomorphic case is used in the discussion of the classical examples.

The zero locus of a nontrivial section of $\omega_{C}^{k}$ on a nonsingular curve $C$ is a $k$-canonical divisor. The moduli space of $k$-canonical divisors is an open set of the proper moduli space of twisted $k$-canonical divisors defined in $\S 6$ for all $k \geqslant 0$. However, our results for $k \neq 1$ are weaker since the associated dimension theory in the case of nonsingular curves is yet to be developed.

\subsection{Related work}

There are several approaches to compactifying the spaces of canonical divisors:

- Janda's approach (which has been discussed briefly above) is motivated by relative Gromov-Witten theory and the proof of Pixton's conjecture for the double ramification cycle [18]. Our moduli of twisted canonical divisors predicts the image of Janda's space under the map to $\overline{\mathcal{M}}_{g, n}$. Because of the pure dimension result of Theorem 3 in the strictly meromorphic case, the push-forward of the virtual class can also be predicted (see the Appendix A).

The purity of dimension in the strictly meromorphic case allows for the approach to Pixton's formula taken in the Appendix A based entirely on classical geometry. The development is unexpected. For example, both the definition and the calculation of the double ramification cycle in [18] make essential use of obstruction theories and virtual fundamental classes.

- Sauvaget and Zvonkine [26] propose a different approach to the compactification of canonical divisors which stays closer to the projectivization of the Hodge bundle

$$
\mathbb{P}(\mathbb{E}) \rightarrow \overline{\mathcal{M}}_{g}
$$

An advantage is the existence of the tautological line $\mathcal{O}(1)$ of the projectivization which is hoped eventually to provide a link to the volume calculations of $[9,10]$. There should also be connections between the recursions for fundamental classes found by Sauvaget and Zvonkine and the conjecture of the Appendix A.

- Twisting related (but not equal) to Definition 1 was studied by Gendron [14]. Chen [4] considered twists in the compact type case motivated by the study of limit linear series (however [4, Proposition 4.23] for stable curves is related to the theory of twists presented here). In further work of Bainbridge et al. [1], the authors study twists and residue conditions for stable curves via analytic methods with the goal of characterizing the closure $\overline{\mathcal{H}}_{g}(\mu)$. 
For the study of the moduli of canonical divisors from the point of Teichmüller dynamics, we refer the reader to [9].

Our paper takes a more naive perspective than the work discussed above. We propose a precise definition of a twisted canonical divisor and take the full associated moduli space seriously as a mathematical object.

\section{Twists of degenerating canonical bundles}

\subsection{Valuative criterion}

Let $\mu=\left(m_{1}, \ldots, m_{n}\right)$ be a vector of zero and pole multiplicities satisfying

$$
\sum_{i=1}^{n} m_{i}=2 g-2 .
$$

By Definition 1,

$$
\tilde{\mathcal{H}}_{g}(\mu) \subset \overline{\mathcal{M}}_{g, n}
$$

is easily seen to be a constructible subset.

Proposition 4. The locus $\widetilde{\mathcal{H}}_{g}(\mu) \subset \overline{\mathcal{M}}_{g, n}$ is Zariski closed.

To prove Proposition 4, we use the valuative criterion. Consider a 1-parameter family of stable $n$-pointed curves over a disk $\Delta$,

$$
\pi: \mathcal{C} \rightarrow \Delta, \quad p_{1}, \ldots, p_{n}: \Delta \rightarrow \mathcal{C},
$$

where the punctured disk $\Delta^{\star}=\Delta \backslash 0$ maps to $\widetilde{\mathcal{H}}_{g}(\mu)$. The sections $p_{i}$ correspond to the markings. We must show the special fiber over $0 \in \Delta$,

$$
\left[C_{0}, p_{1}, \ldots, p_{n}\right]
$$

also lies in $\tilde{\mathcal{H}}_{g}(\mu)$.

After possibly shrinking $\Delta$, the topological type of the fibers over $\Delta^{\star}$ may be assumed to be constant. After base change, we may assume there is no monodromy in the components of the fibers over $\Delta^{\star}$. Finally, after further shrinking, we may assume the twist $I$ guaranteed by Definition 1 for each fiber over $\Delta^{\star}$ is the same.

Since the topological type and the twist $I$ is the same over $\Delta^{\star}$, the structures

$$
\operatorname{Irr}\left(C_{\zeta}\right), \quad \operatorname{Irr}\left(C_{\zeta}\right)^{\sim}, \quad \Gamma_{I}\left(C_{\zeta}\right)
$$

do not vary as the fiber $C_{\zeta}$ varies over $\zeta \in \Delta^{\star}$.

The basic nodes of the special fiber $C_{0}$ are of two types: basic nodes smoothed by the family $\pi$ and basic nodes not smoothed by the family. The unsmoothed basic nodes correspond to basic nodes of $C_{\zeta \neq 0}$, so the twist $I$ already assigns integers to the unsmoothed basic nodes of $C_{0}$. However, we must assign twists to the basic nodes of $C_{0}$ which are smoothed by $\pi$.

For $\zeta \in \Delta^{\star}$, consider a vertex $v \in \Gamma_{I}\left(C_{\zeta}\right)$ which corresponds (by taking the union of the irreducible components in $\sim$-equivalence class $v$ ) to a connected subcurve $C_{\zeta}^{v}$. As $\zeta$ varies, an associated family

$$
\mathcal{C}^{v} \rightarrow \Delta^{\star}
$$


is defined with closure in $\mathcal{C}$ given by

$$
\pi^{v}: \overline{\mathcal{C}}^{v} \rightarrow \Delta
$$

The markings which lie on $\overline{\mathcal{C}}^{v}$ yield sections

$$
p_{1_{v}}, \ldots, p_{x_{v}}: \Delta \rightarrow \overline{\mathcal{C}}^{v}
$$

The nodes connecting $C_{\zeta}^{v}$ to the complement in $C_{\zeta}$ yield further sections

$$
q_{1_{v}}, \ldots, q_{y_{v}}: \Delta \rightarrow \overline{\mathcal{C}}^{v}
$$

at which the twist $I$ is not zero.

By the definition of a twisted canonical divisor, we have

$$
\omega_{C_{\zeta}^{v}} \simeq \mathcal{O}_{C_{\zeta}^{v}}\left(\sum_{i=1}^{x} m_{i_{v}} p_{i_{v}}-\sum_{j=1}^{y}\left(I\left(q_{j_{v}}, v\right)+1\right) q_{j, v}\right) .
$$

Hence, the curve

$$
\left[C_{\zeta}^{v}, p_{1_{v}}, \ldots, p_{x_{v}}, q_{1_{v}}, \ldots, q_{y_{v}}\right]
$$

is a twisted canonical divisor for the vector

$$
\left(m_{1_{v}}, \ldots, m_{x_{v}},-I\left(q_{1_{v}}, v\right)-1, \ldots,-I\left(q_{y_{v}}, v\right)-1\right) .
$$

In $\S 1.2$ below, we show the fiber of

$$
\pi^{v}: \overline{\mathcal{C}}^{v} \rightarrow \Delta, \quad p_{1_{v}}, \ldots, p_{x_{v}}, q_{1_{v}}, \ldots, q_{y_{v}}: \Delta \rightarrow \mathcal{C},
$$

over $0 \in \Delta$ is a twisted canonical divisor (with nonzero twists only at basic nodes of $\bar{C}_{0}^{v}$ which are smoothed by the family $\pi^{v}$ ).

By considering all the vertices $v \in \Gamma_{I}\left(C_{\zeta \neq 0}\right)$, we define twists at all basic nodes of the special fiber $C_{0}$ which are smoothed by $\pi$. We now have defined twists at all basic nodes of $C_{0}$,

$$
I_{0}: \widetilde{\mathrm{BN}}\left(C_{0}\right) \rightarrow \mathbb{Z}
$$

Via these twists, we easily see

$$
\left[C_{0}, p_{1}, \ldots, p_{n}\right]
$$

is a twisted canonical divisor. Checking the balancing, vanishing, sign, and transitivity conditions is straightforward:

- Balancing holds for $I_{0}$ by construction.

- The vanishing, sign, and transitivity for $I_{0}$ are all implied by the respective conditions for $I$ and for the twists constructed on $\overline{\mathcal{C}}^{v}$.

- The required isomorphism of line bundles on the partial normalization

$$
v: C_{0, I_{0}} \rightarrow C_{0}
$$

determined by $I_{0}$ is a consequence of corresponding isomorphisms on the partial normalizations of $\overline{\mathcal{C}}_{0}^{v}$ determined by the twists. 


\subsection{Generically untwisted families}

In $\S 1.1$, we have reduced the analysis of the valuative criterion to the generically untwisted case.

Let $\mu=\left(m_{1}, \ldots, m_{n}\right)$ be a vector of zero and pole multiplicities satisfying

$$
\sum_{i=1}^{n} m_{i}=2 g-2
$$

Let

$$
\pi: \mathcal{C} \rightarrow \Delta, \quad p_{1}, \ldots, p_{n}: \Delta \rightarrow \mathcal{C}
$$

be a 1-parameter family of stable $n$-pointed curves for which we have an isomorphism

$$
\omega_{C_{\zeta}} \stackrel{\sim}{=} \mathcal{O}_{C_{\zeta}}\left(\sum_{i=1}^{n} m_{i} p_{i}\right)
$$

for all $\zeta \in \Delta^{\star}$. We show the special fiber over $0 \in \Delta$,

$$
\left[C_{0}, p_{1}, \ldots, p_{n}\right]
$$

is a twisted canonical divisor with respect to $\mu$ via a twist

$$
I_{0}: \widetilde{\mathrm{BN}}\left(C_{0}\right) \rightarrow \mathbb{Z}
$$

supported only on the basic nodes of $C_{0}$ which are smoothed by the family $\pi$.

The total space of the family $\mathcal{C}$ has (at worst) $A_{r}$-singularities at the nodes of $C_{0}$ which are smoothed by $\pi$. Let

$$
\widetilde{\mathcal{C}} \stackrel{\epsilon}{\longrightarrow} \mathcal{C} \stackrel{\pi}{\longrightarrow} \Delta
$$

be the crepant resolution (via chains of (-2)-curves) of all singularities occurring at the smoothed nodes of $C_{0}$. The line bundles

$$
\epsilon^{*} \omega_{\pi} \quad \text { and } \quad \epsilon^{*} \mathcal{O}_{\mathcal{C}}\left(\sum_{i=1}^{n} m_{i} p_{i}\right)
$$

on $\widetilde{\mathcal{C}}$ are isomorphic over $\Delta^{\star}$ by (2). Therefore, the bundles (3) differ by a Cartier divisor $\mathcal{O}_{\widetilde{\mathcal{C}}}(T)$ on $\widetilde{\mathcal{C}}$ satisfying the following properties:

(i) $\mathcal{O}_{\widetilde{\mathcal{C}}}(T)$ is supported over $0 \in \Delta$,

(ii) $\mathcal{O}_{\widetilde{\mathcal{C}}}(T)$ restricts to the trivial line bundle on every exceptional $(-2)$-curve of the resolution $\epsilon$.

By property (i), the Cartier divisor $\mathcal{O}_{\widetilde{\mathcal{C}}}(T)$ must be a sum of irreducible components of the fiber $\widetilde{C}_{0}$ of $\widetilde{\mathcal{C}}$ over $0 \in \Delta$, that is,

$$
T=\sum_{D \in \operatorname{Irr}\left(\widetilde{C}_{0}\right)} \gamma_{D} \cdot D, \quad \text { with } \gamma_{D} \in \mathbb{Z}
$$


The irreducible components of $\widetilde{C}_{0}$ correspond $^{4}$ to the irreducible components of $C_{0}$ together with all the exceptional $(-2)$-curves,

$$
\operatorname{Irr}\left(\widetilde{C}_{0}\right)=\operatorname{Irr}\left(C_{0}\right) \cup\left\{E_{i}\right\}
$$

The Cartier property implies that

$$
\gamma_{D}=\gamma_{D^{\prime}}
$$

for distinct components $D, D^{\prime} \in \operatorname{Irr}\left(C_{0}\right)$ which intersect in at least 1 node of $C_{0}$ which is not smoothed by $\pi$.

Let $E_{1} \cup \cdots \cup E_{r} \subset \widetilde{C}_{0}$ be the full exceptional chain of (-2)-curves for the resolution of an $A_{r}$-singularity of $\mathcal{C}$ corresponding to a node $q \in C_{0}$ which is smoothed by $\pi$. We have the following data:

- $E_{1}$ intersects the irreducible component $D \subset \widetilde{C}_{0}$,

- $E_{r}$ intersects the irreducible component $D^{\prime} \subset \widetilde{C}_{0}$;

see Figure 1. By property (ii), we find $r$ equations obtained from the triviality of $\mathcal{O}_{\widetilde{\mathcal{C}}}(T)$ on each subcurve $E_{i}$ :

$$
2 \gamma_{E_{1}}=\gamma_{D}+\gamma_{E_{2}}, \ldots, 2 \gamma_{E_{i}}=\gamma_{E_{i-1}}+\gamma_{E_{i+1}}, \ldots, 2 \gamma_{E_{r}}=\gamma_{E_{r-1}}+\gamma_{D^{\prime}}
$$

The equations (4) are uniquely solvable ${ }^{5}$ in the variables $\gamma_{E_{1}}, \ldots, \gamma_{E_{r}}$ in terms of $\gamma_{D}$ and $\gamma_{D^{\prime}}$. If $\gamma_{D}=\gamma_{D^{\prime}}$, then the unique solution is

$$
\gamma_{E_{i}}=\gamma_{D} \text { for all } i
$$

The solution (5) is always the case when $q$ is a nonbasic node (since then $D=D^{\prime}$ ).

If $q$ is a basic node and $\gamma_{D} \neq \gamma_{D^{\prime}}$, then equations (4) imply the values of $\gamma_{E_{i}}$ are uniformly spaced and lie strictly between $\gamma_{D}$ and $\gamma_{D^{\prime}}$. For example, if $\gamma_{D}=3$ and $\gamma_{D^{\prime}}=9$ and $r=2$, we have

$$
3<\gamma_{E_{1}}=5<\gamma_{E_{2}}=7<9
$$

The equations imply

$$
-\gamma_{D}+\gamma_{E_{1}}=-\gamma_{E_{r}}+\gamma_{D^{\prime}}
$$

If $q \in \mathrm{BN}\left(C_{0}\right)$ is a basic node, then we assign the twists

$$
I_{0}(q, D)=-\gamma_{D}+\gamma_{E_{1}}, \quad I_{0}\left(q, D^{\prime}\right)=-\gamma_{D^{\prime}}+\gamma_{E_{r}} .
$$

If there are no (-2)-curves over $q \in C_{0}$, then

$$
I_{0}(q, D)=-\gamma_{D}+\gamma_{D^{\prime}}, \quad I_{0}\left(q, D^{\prime}\right)=-\gamma_{D^{\prime}}+\gamma_{D} .
$$

Lemma 5. The assignment $I_{0}: \mathrm{BN}\left(C_{0}\right) \rightarrow \mathbb{Z}$ defined above satisfies the balancing, vanishing, sign, and transitivity conditions.

\footnotetext{
${ }^{4}$ The irreducible components of $C_{0}$ maybe be partially normalized in $\widetilde{C}_{0}$. We denote the strict transform of $D \in \operatorname{Irr}\left(C_{0}\right)$ also by $D \in \operatorname{Irr}\left(\widetilde{C}_{0}\right)$.

${ }^{5}$ The unique solution yields $\gamma_{E_{i}} \in \mathbb{Q}$. For solution $\gamma_{E_{i}} \in \mathbb{Z}, r+1$ must divide $-\gamma_{D}+\gamma_{D^{\prime}}$.
} 


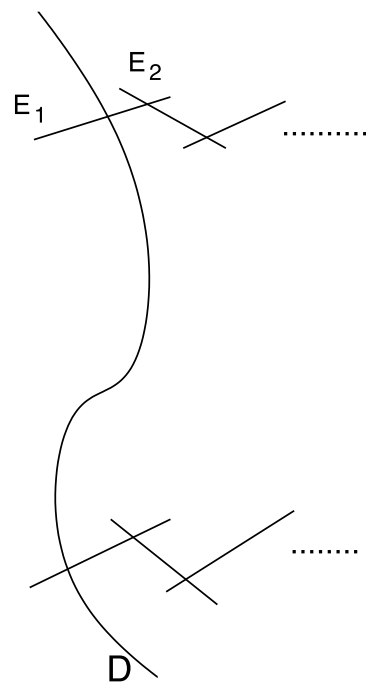

$\widetilde{\mathrm{C}}_{0}$

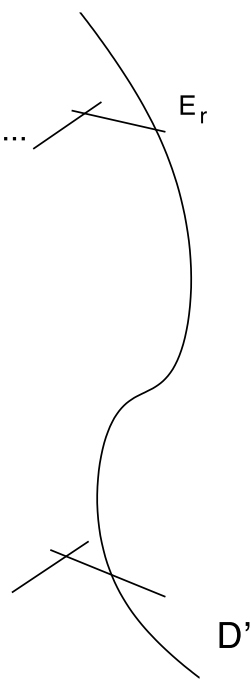

$\widetilde{C}_{0}$

Figure 1. The curve $\widetilde{C}_{0}$.

Proof. As usual, the balancing condition holds by construction (6). We index the components $D \in \operatorname{Irr}\left(C_{0}\right)$ by the integer $\gamma_{D}$,

$$
\gamma: \operatorname{Irr}\left(C_{0}\right) \rightarrow \mathbb{Z}, \quad D \mapsto \gamma_{D} .
$$

The equivalence relation $\sim$ defined by $I_{0}$ is easily understood: the equivalence classes are exactly maximal connected subcurves of $C_{0}$ for which the value of $\gamma$ is constant. The basic nodes of $C_{0}$ lying entirely within such a subcurve have twist 0 by (5). The basic nodes of $C_{0}$ connecting different equivalence classes have nonzero twist by the uniform spacing property of the solutions to (4). The vanishing condition is therefore established. The sign condition follows again from the uniform spacing property. A directed edge in $\operatorname{Irr}\left(C_{0}\right)^{\sim}$ points in the direction of higher $\gamma$ values, so transitivity is immediate.

Finally, in order to show that the special fiber of $\pi$,

$$
\left[C_{0}, p_{1}, \ldots, p_{n}\right],
$$

is a twisted canonical divisor (with respect to $\mu$ and $I_{0}$ ), we must check the isomorphism

$$
\nu^{*} \mathcal{O}_{C_{0}}\left(\sum_{i=1}^{n} m_{i} p_{i}\right) \cong v^{*}\left(\omega_{C_{0}}\right) \otimes \mathcal{O}_{C_{0, I_{0}}}\left(\sum_{q \in \mathrm{N}_{I}} I\left(q, D_{q}^{\prime}\right) \cdot q^{\prime}+I\left(q, D_{q}^{\prime \prime}\right) \cdot q^{\prime \prime}\right)
$$

on the partial normalization

$$
C_{0, I_{0}} \rightarrow C_{0} \text {. }
$$

A connected component $C_{0, I_{0}}^{v}$ of the partial normalization $C_{0, I_{0}}$ corresponds to a vertex $v \in \operatorname{Irr}\left(C_{0}\right)^{\sim}$. A node of $C_{0, I_{0}}^{v}$, necessarily untwisted by $I_{0}$, corresponds to a chain of 
(-2)-curves of $\widetilde{\mathcal{C}_{0}}$. After inserting these corresponding chains of rational components at the nodes of $C_{0, I_{0}}^{v}$, we obtain a curve $\widetilde{C}_{0, I_{0}}^{v}$ with canonical maps

$$
C_{0, I_{0}}^{v} \leftarrow \widetilde{C}_{0, I_{0}}^{v} \rightarrow \tilde{\mathcal{C}}_{0}
$$

The left map of (8) contracts the added rational components and the right map of (8) is the inclusion. The isomorphism (7) on $C_{0, I_{0}}^{v}$ follows directly from the isomorphism

$$
\epsilon^{*} \mathcal{O}_{\mathcal{C}}\left(\sum_{i=1}^{n} m_{i} p_{i}\right) \stackrel{\simeq}{=} \epsilon^{*} \omega_{\pi} \otimes \mathcal{O}_{\widetilde{\mathcal{C}}^{(T)}}
$$

on $\widetilde{C}$ after pull-back via the right map of (8) and push-forward via the left map of (8). The proof of Proposition 4 is complete.

\subsection{Smoothings}

Let $\mu=\left(m_{1}, \ldots, m_{n}\right)$ be a vector of zero and pole multiplicities satisfying

$$
\sum_{i=1}^{n} m_{i}=2 g-2
$$

Let

$$
\left[C, p_{1}, \ldots, p_{n}\right] \in \widetilde{\mathcal{H}}_{g}(\mu) \subset \overline{\mathcal{M}}_{g, n},
$$

be a twisted canonical divisor via a twist

$$
I: \widetilde{\mathrm{BN}}(C) \rightarrow \mathbb{Z}
$$

Lemma 6. There exists a 1-parameter family

$$
\pi: \mathcal{C} \rightarrow \Delta, \quad p_{1}, \ldots, p_{n}: \Delta \rightarrow \mathcal{C}
$$

of stable $n$-pointed curves and a line bundle

$$
\mathcal{L} \rightarrow \mathcal{C}
$$

satisfying the following properties:

(i) There is an isomorphism of n-pointed curves

$$
\pi^{-1}(0) \stackrel{\simeq}{=}\left[C, p_{1}, \ldots, p_{n}\right]
$$

under which

$$
\mathcal{L}_{0} \stackrel{\sim}{=} \mathcal{O}_{C}\left(\sum_{i=1}^{n} m_{i} p_{i}\right)
$$

(ii) The generic fiber

$$
C_{\zeta}=\pi^{-1}(\zeta), \quad \zeta \in \Delta^{\star}
$$

is a nonsingular curve and

$$
\mathcal{L}_{\zeta} \stackrel{\sim}{=} \omega_{C_{\zeta}}
$$


Lemma 6 shows the line bundle associated to a twisted canonical divisor can always be smoothed to a canonical line bundle on a nonsingular curve.

Proof. The twist $I$ determines connected subcurves $C_{v} \subset C$ associated to equivalence classes $v \in \operatorname{Irr}(C)^{\sim}$. Let

$$
\pi: \mathcal{C} \rightarrow \Delta, \quad p_{1}, \ldots, p_{n}: \Delta \rightarrow \mathcal{C}
$$

be a smoothing of the special fiber $\left[C, p_{1}, \ldots, p_{n}\right]$. We can construct a line bundle

$$
\mathcal{L} \cong \omega_{\pi}\left(\sum_{v \in \operatorname{Irr}(C)^{\sim}} \gamma_{v} \cdot\left[C_{v}\right]\right)
$$

for integers $\gamma_{v}$. The restriction of $\mathcal{L}$ determines a twist

$$
I^{\gamma}: \widetilde{\mathrm{BN}}(C) \rightarrow \mathbb{Z}
$$

by the following rule: if $q \in C_{v} \cap C_{w}$, then

$$
I^{\gamma}\left(q, C_{v}\right)=-\gamma_{v}+\gamma_{w} .
$$

In all other cases, $I^{\gamma}$ vanishes. An immediate issue is whether

$$
\gamma: \operatorname{Irr}(C)^{\sim} \rightarrow \mathbb{Z} \quad v \mapsto \gamma_{v}
$$

can be chosen so

$$
I^{\gamma}=I
$$

Unfortunately, equality (9) may be impossible to satisfy. A simple obstruction is the following: if

$$
q, q^{\prime} \in \mathrm{BN}(C)
$$

both lie in the intersection of the subcurves $C_{v}$ and $C_{w}$, then

$$
I^{\gamma}\left(q, C_{v}\right)=I^{\gamma}\left(q^{\prime}, C_{w}\right) .
$$

So if $I\left(q, C_{v}\right) \neq I\left(q^{\prime}, C_{w}\right)$, then $I^{\gamma} \neq I$ for all $\gamma$. In order to satisfy (9), we destabilize the special fiber $C$ by adding chains of rational components at the nodes of $\operatorname{BN}(C)$ at which $I$ is supported.

Let $\Gamma_{I}(C)$ be the directed graph associated to $I$ with vertex $\operatorname{set} \operatorname{Irr}(C)^{\sim}$. For $v \in \operatorname{Irr}(C)^{\sim}$, we define $\operatorname{depth}(v)$ to be the length of the longest chain of directed edges which ends in $v$. By the transitivity condition for the twist $I, \operatorname{depth}(v)$ is finite and nonnegative. ${ }^{6}$ Let

$$
M_{I}=\prod_{(q, D) \in \widetilde{\operatorname{BN}}(C), I(q, D)>0} I(q, D) .
$$

We define $\gamma_{v} \in \mathbb{Z}$ by

$$
\gamma_{v}=\operatorname{depth}(v) \cdot M_{I}
$$

${ }^{6}$ The depth of $v$ may be 0. 
Let $q \in \mathrm{BN}(C)$ be a node lying in the intersection

$$
q \in C_{v} \cap C_{w} \quad \text { with } I\left(q, C_{v}\right)>0 .
$$

Since $\operatorname{depth}(v)<\operatorname{depth}(w)$ and

$$
M_{I} \mid-\gamma_{v}+\gamma_{w} \text { we have } I\left(q, C_{v}\right) \mid-\gamma_{v}+\gamma_{w} .
$$

We add a chain of

$$
\frac{-\gamma_{v}+\gamma_{w}}{I\left(q, C_{v}\right)}-1
$$

destabilizing rational curve at each such node $q$. For each rational curve $P_{i}^{q}$ in the chain

$$
P_{1}^{q} \cup \cdots \cup P_{\frac{-\gamma v+\gamma_{w}}{I\left(q, C_{v}\right)}-1}^{q},
$$

we define $\gamma_{P_{i}^{q}}=\gamma_{v}+i \cdot I\left(q, C_{v}\right)$.

The result is a new curve $\widetilde{C}$ with a map

$$
\widetilde{C} \rightarrow C
$$

contracting the added chains. Moreover, for a 1-parameter family

$$
\tilde{\pi}: \widetilde{\mathcal{C}} \rightarrow \Delta, \quad p_{1}, \ldots, p_{n}: \Delta \rightarrow \widetilde{\mathcal{C}}
$$

smoothing the special fiber $\left[\widetilde{C}, p_{1}, \ldots, p_{n}\right]$, we construct a line bundle ${ }^{7}$

$$
\tilde{\mathcal{L}} \cong \omega_{\tilde{\pi}}\left(\sum_{v \in \operatorname{lrr}(C)^{\sim}} \gamma_{v} \cdot\left[C_{v}\right]+\sum_{q \in \operatorname{BN}(C)} \sum_{i=1}^{\frac{\gamma_{w}-\gamma_{v}}{I\left(q, C_{v}\right)}-1} \gamma_{P_{i}^{q}} \cdot\left[P_{i}^{q}\right]\right) .
$$

The line bundle $\widetilde{\mathcal{L}}$ satisfies several properties:

(i) $\left.\widetilde{\mathcal{L}}\right|_{P_{i}^{q}}$ is trivial,

(ii) for every $v \in \operatorname{Irr}(C)^{\sim}$,

$$
\left.\left.\widetilde{\mathcal{L}}\right|_{C_{v}} \stackrel{\sim}{=} \omega_{C}\right|_{C_{v}} \otimes \mathcal{O}_{C_{v}}\left(\sum_{q \in \mathrm{N}_{I}} I\left(q, C_{v}\right) \cdot q\right) \stackrel{\simeq}{=} \mathcal{O}_{C_{v}}\left(\sum_{p_{i} \in C_{v}} m_{i} p_{i}\right),
$$

(iii) for $\zeta \in \Delta^{\star}, \widetilde{\mathcal{L}}_{\zeta} \stackrel{\sim}{=} \omega_{\widetilde{C}_{\zeta}}$.

We can contract the extra rational components $P_{i}^{q}$ in the special fiber of $\widetilde{\mathcal{C}}$ to obtain 1 -parameter family of stable $n$-pointed curves

$$
\pi: \mathcal{C} \rightarrow \Delta, \quad p_{1}, \ldots, p_{n}: \Delta \rightarrow \mathcal{C}
$$

which smooths $\left[C, p_{1}, \ldots, p_{n}\right]$. By (i), the line bundle $\widetilde{\mathcal{L}}$ descends to

$$
\mathcal{L} \rightarrow \mathcal{C} .
$$

${ }^{7}$ The subcurve $C_{v} \subset C$ corresponds to a subcurve $C_{v} \subset \widetilde{C}$ by strict transformation. 
By (ii), for every $v \in \operatorname{Irr}(C)^{\sim}$,

$$
\left.\mathcal{L}\right|_{C_{v}} \stackrel{\sim}{=} \mathcal{O}_{C_{v}}\left(\sum_{p_{i} \in C_{v}} m_{i} p_{i}\right) .
$$

The isomorphisms (12) after restriction to the subcurves do not quite imply the required isomorphism

$$
\mathcal{L}_{0}=\left.\mathcal{L}\right|_{C} \stackrel{\sim}{=} \mathcal{O}_{C}\left(\sum_{i=1}^{n} m_{i} p_{i}\right)
$$

because there are additional $h^{1}\left(\Gamma_{I}\right)$ factors of $\mathbb{C}^{*}$ in the Picard variety of $C$. However, these $\mathbb{C}^{*}$-factors for

$$
\left.\mathcal{L}\right|_{C} \quad \text { and } \quad \mathcal{O}_{C_{v}}\left(\sum_{i=1}^{n} m_{i} p_{i}\right)
$$

can be matched by correctly choosing the smoothing parameters at the nodes of $\widetilde{C}$ in the original family (11).

\section{Dimension estimates}

\subsection{Estimates from above}

Let $\mu=\left(m_{1}, \ldots, m_{n}\right)$ be a vector ${ }^{8}$ of zero and pole multiplicities satisfying

$$
\sum_{i=1}^{n} m_{i}=2 g-2 .
$$

The boundary

$$
\partial \overline{\mathcal{M}}_{g, n} \subset \overline{\mathcal{M}}_{g, n}
$$

is the divisor parameterizing stable $n$-pointed curves with at least one node. We estimate from above the dimension of irreducible components of $\tilde{\mathcal{H}}_{g}(\mu)$ supported in the boundary.

Proposition 7. Every irreducible component of $\widetilde{\mathcal{H}}_{g}(\mu)$ supported entirely in the boundary of $\overline{\mathcal{M}}_{g, n}$ has dimension at most $2 g-3+n$.

Proof. Let $Z \subset \widetilde{\mathcal{H}}_{g}(\mu)$ be an irreducible component supported entirely in $\partial \overline{\mathcal{M}}_{g, n}$. Let $\Gamma_{Z}$ be the dual graph of $C$ for the generic element

$$
\left[C, p_{1}, \ldots, p_{n}\right] \in Z .
$$

The dual graph ${ }^{9}$ consists of vertices (with genus assignment), edges, and legs (corresponding to the markings):

$$
\Gamma_{Z}=\left(\mathrm{V}, \mathrm{E}, \mathrm{L}, \mathrm{g}: \mathrm{V} \rightarrow \mathbb{Z}_{\geqslant 0}\right)
$$

\footnotetext{
${ }^{8}$ For the dimension estimates here, we consider all $\mu$. No assumptions on the parts are made.

${ }^{9} \mathrm{~A}$ refined discussion of dual graphs via half-edges is required in the Appendix A. Here, a simpler treatment is sufficient.
} 
Each vertex $v \in \mathrm{V}$ corresponds to an irreducible component of $C$. The valence $\mathrm{n}(v)$ counts both half-edges and legs incident to $v$. The genus formula is

$$
g-1=\sum_{v \in \mathrm{V}}(\mathrm{g}(v)-1)+|\mathrm{E}| .
$$

Since $Z$ is supported in the boundary, $\Gamma_{Z}$ must have at least one edge. We estimate from above the dimension of the moduli of twisted canonical divisors in $\overline{\mathcal{M}}_{g, n}$ with dual graph exactly $\Gamma_{Z}$. Let $v \in \mathrm{V}$ be a vertex corresponding to the moduli space $\mathcal{M}_{\mathrm{g}(v), \mathrm{n}(v)}$. The dimension of the moduli of the canonical divisors on the component corresponding to $v$ is bounded from above by

$$
2 \mathrm{~g}(v)-2+\mathrm{n}(v)
$$

Here, we have used the dimension formula for the locus of canonical divisors with prescribed zero multiplicities [25]. The strictly meromorphic case has lower dimension (see $\S 0.1$ ).

Summing over the vertices yields a dimension bound for $Z$ :

$$
\begin{aligned}
\operatorname{dim} Z & \leqslant \sum_{v \in \mathrm{V}} 2 \mathrm{~g}(v)-2+\mathrm{n}(v) \\
& =2 \sum_{v \in \mathrm{V}}(\mathrm{g}(v)-1)+2|\mathrm{E}|+n \\
& =2 g-2+n .
\end{aligned}
$$

The result falls short of the claim of the proposition by 1 .

We improve the bound (15) by the following observation. The dual graph $\Gamma_{Z}$ must have at least one edge $e$ since $Z$ is supported in the boundary:

- If the edge $e$ corresponds to a nonbasic node, then the vertex incident to $e$ is allowed a pole at the two associated nodal points.

- If the edge $e$ corresponds to a basic node, there must be at least one vertex $v \in \mathrm{V}$ incident to $e$ which carries meromorphic differentials.

In either case, we can apply the stronger bound

$$
2 \mathrm{~g}(v)-3+\mathrm{n}(v) .
$$

Since we are guaranteed at least one application of (16), we conclude

$$
\operatorname{dim} Z \leqslant 2 g-3+n
$$

which is better than (15) by 1 .

\subsection{Star graphs}

Let $Z \subset \widetilde{\mathcal{H}}_{g}(\mu)$ be an irreducible component supported entirely in $\partial \overline{\mathcal{M}}_{g, n}$. By the proof of Proposition 7 , if $\Gamma_{Z}$ has for every twist $I$ at least two vertices corresponding to meromorphic differentials, then

$$
\operatorname{dim} Z \leqslant 2 g-4+n
$$


The dual graphs $\Gamma_{Z}$ with a least one edge (since $Z$ is supported in the boundary) and carrying a twist $I$ with only one vertex corresponding to meromorphic differentials are easy to classify. The dual graph $\Gamma_{Z}$ must be a star:

- The vertices $\left\{v_{0}, v_{1}, \ldots, v_{k}\right\}$ of $\Gamma_{Z}$ consist of a center vertex $v_{0}$ and outlying vertices $\left\{v_{1}, \ldots, v_{k}\right\}$.

- The edges of $\Gamma_{Z}$ are of two types:

(i) self-edges at $v_{0}$,

(ii) edges (possibly multiple) connecting the center $v_{0}$ to the outlying vertices.

- The parts ${ }^{10}$ of $\mu$ are distributed to the vertices with all negative parts distributed to the center $v_{0}$.

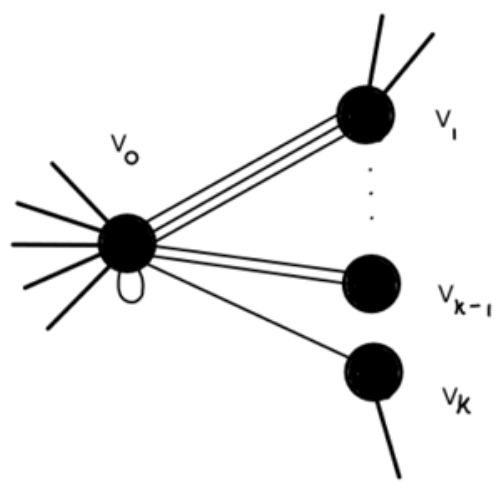

A twist $I$ which orients every edge of type (ii) as outgoing from $v_{0}$ yields meromorphic differentials at $v_{0}$ only. The self-edges at $v_{0}$ correspond to nonbasic nodes and cannot be twisted.

\subsection{Twisted canonical bundles}

We have defined a twisted canonical divisor in $\S 0.4$. A twisted canonical divisor determines a section (up to scale) of a twisted canonical bundle on $C$.

Definition 8. A line bundle $L$ on a connected nodal curve $C$ is twisted canonical if there exists a twist

$$
I: \widetilde{\mathrm{BN}}(C) \rightarrow \mathbb{Z}
$$

for which there is an isomorphism

$$
v^{*} L \stackrel{\sim}{=} v^{*}\left(\omega_{C}\right) \otimes \mathcal{O}_{C_{I}}\left(\sum_{q \in \mathrm{N}_{I}} I\left(q, D_{q}^{\prime}\right) \cdot q^{\prime}+I\left(q, D_{q}^{\prime \prime}\right) \cdot q^{\prime \prime}\right)
$$

on the partial normalization $\mu: C_{I} \rightarrow C$.

${ }^{10}$ The parts of $\mu$ correspond to the markings. 
The limits of twisted canonical line bundles are also twisted canonical. The proof has already been given as a step in the proof of the properness of $\widetilde{\mathcal{H}}_{g}(\mu)$ in $\S 1$. We state the result as a lemma.

Lemma 9. Let $\pi: \mathcal{C} \rightarrow \Delta$ be a flat family of connected nodal curves, and let

$$
\mathcal{L} \rightarrow \mathcal{C}
$$

be a line bundle. If $L_{\zeta} \rightarrow C_{\zeta}$ is a twisted canonical line bundle for all $\zeta \in \Delta^{\star}$, then

$$
L_{0} \rightarrow C_{0}
$$

is also a twisted canonical line bundle.

The definition of a twisted canonical line bundle does not specify a twist. Only the existence of a twist is required. However, there are only finitely many possible twists.

Lemma 10. If $L \rightarrow C$ is a twisted canonical line bundle, there are only finitely many twists

$$
I: \widetilde{\mathrm{BN}}(C) \rightarrow \mathbb{Z}
$$

for which there exists an isomorphism

$$
v^{*} L \cong v^{*}\left(\omega_{C}\right) \otimes \mathcal{O}_{C_{I}}\left(\sum_{q \in \mathrm{N}_{I}} I\left(q, D_{q}^{\prime}\right) \cdot q^{\prime}+I\left(q, D_{q}^{\prime \prime}\right) \cdot q^{\prime \prime}\right)
$$

on the partial normalization $\mu: C_{I} \rightarrow C$.

Proof. Let $I$ be a twist for which the above isomorphism holds. By definition, $I$ must satisfy the balancing, vanishing, sign, and transitivity conditions of $\S 0.3$. Since there are no directed loops in the graph $\Gamma_{I}(C)$, there must be a vertex $v$ with only outgoing arrows. Let

$$
C_{v} \subset C
$$

be the associated subcurve. The twist $I$ is always positive on the nodes $\mathrm{N}_{I}$ incident to the subcurve $C_{v}$ (on the side of $C_{v}$ ). Since the degrees of $\left.v^{*}(L)\right|_{C_{v}}$ and $\left.v^{*}\left(\omega_{C}\right)\right|_{C_{v}}$ are determined, we obtain a bound on the twists of $I$ on the nodes $\mathrm{N}_{I}$ incident to $C_{v}$ : only finitely many values for $I$ on these nodes are permitted.

Next, we find a vertex $v^{\prime} \in \Gamma_{I}(C)$ which has only outgoing arrows (except from possibly $v$ ). Repeating the above argument easily yields the required finiteness statement for $I$.

\subsection{Estimates from below}

Let $\mu=\left(m_{1}, \ldots, m_{n}\right)$ be a vector of zero and pole multiplicities satisfying

$$
\sum_{i=1}^{n} m_{i}=2 g-2
$$

We now prove a lower bound on the dimension of irreducible components of $\widetilde{\mathcal{H}}_{g}(\mu)$. 
Proposition 11. Every irreducible component of $\widetilde{\mathcal{H}}_{g}(\mu)$ has dimension at least $2 g-3+n$. Proof. Let $\left[C, p_{1}, \ldots, p_{n}\right] \in \widetilde{\mathcal{H}}_{g}(\mu)$ be a stable $n$-pointed curve. Let

$$
L \stackrel{\sim}{=} \mathcal{O}_{C}\left(\sum_{i=1}^{n} m_{i} p_{i}\right) \rightarrow C
$$

be the associated twisted canonical bundle. Let

$$
m_{1}, \ldots, m_{k}
$$

be the negative parts of $\mu$ (if there are any). Consider the $k$-pointed nodal curve

$$
\left[C, p_{1}, \ldots, p_{k}\right]
$$

obtained by dropping ${ }^{11}$ the markings $p_{k+1}, \ldots, p_{n}$. Let $r_{1}$ and $r_{2}$ denote the number of rational components of $\left[C, p_{1}, \ldots, p_{k}\right]$ with exactly 1 and 2 special points, respectively. To kill the automorphisms of these unstable components, we add new markings

$$
q_{1}, \ldots, q_{2} r_{1}+r_{2}
$$

to the curve $C$ (away from $p_{1}, \ldots, p_{k}$ and away from the nodes):

- we add two $q$ 's on each component with 1 special point,

- we add one $q$ on each component with 2 special points.

The result

$$
\left[C, p_{1}, \ldots, p_{k}, q_{1}, \ldots, q_{2 r_{1}+r_{2}}\right]
$$

is a stable pointed curve.

Let $\mathcal{V}$ be the nonsingular versal deformation space of the $k+2 r_{1}+r_{2}$-pointed curve (17),

$$
\operatorname{dim} \mathcal{V}=\operatorname{dim} \operatorname{Def}\left(\left[C, p_{1} \ldots, p_{k}, q_{1}, \ldots, q_{2 r_{1}+r_{2}}\right]\right)=3 g-3+k+2 r_{1}+r_{2}
$$

There is a universal curve $\mathrm{e}^{12}$

$$
\pi: \mathcal{C} \rightarrow \mathcal{V}, \quad p_{1}, \ldots, p_{k}: \mathcal{V} \rightarrow \mathcal{C}
$$

We consider the relative moduli space ${ }^{13}$ of degree $2 g-2$ line bundles on the fibers of $\pi$,

$$
\epsilon: \mathcal{B} \rightarrow \mathcal{V}
$$

Let $\mathcal{V}^{\star} \subset \mathcal{V}$ be the locus of nonsingular curves in the versal deformation space, and let

$$
\mathcal{B}^{\star} \rightarrow \mathcal{V}^{\star}
$$

be the relative Jacobian of degree $2 g-2$. Let

$$
\mathcal{W}^{\star} \subset \mathcal{B}^{\star}
$$

\footnotetext{
${ }^{11}$ We do not contract unstable components.

${ }^{12}$ We will not use the $q$-sections.

${ }^{13}$ The Quot scheme parameterization of line bundles can be used to avoid the separation issues of the moduli of line bundles. The dimension calculus is parallel.
} 
be the codimension $g$ locus in the universal Jacobian defined fiberwise by the relative canonical bundle $\omega_{\pi}$. Let $\mathcal{W}$ be the closure of $\mathcal{W}^{\star}$ in $\mathcal{B}$. By Lemma 9 , every point of $\mathcal{W}$ parameterizes a twisted canonical bundle. By Lemma 6 , the line bundle $L$ lies in $\mathcal{W}$ over the special fiber $[C] \in \mathcal{V}$. The dimension of $\mathcal{W}$ is

$$
\operatorname{dim} \mathcal{W}=\operatorname{dim} \mathcal{B}^{\star}-g=3 g-3+k+2 r_{1}+r_{2} .
$$

Let $\left[C^{\prime}, L^{\prime}\right] \in \mathcal{W}$ be a pair where $\left[C^{\prime}\right] \in \mathcal{V}$ and

$$
L^{\prime} \rightarrow C^{\prime}
$$

is a twisted canonical bundle. A good section of $L^{\prime}\left(-\sum_{i=1}^{k} m_{i} p_{i}\right)$ on $C^{\prime}$ is section $s$ satisfying the following properties:

- $s$ does not vanish at $p_{1}, \ldots, p_{k}$,

- $s$ does not vanish at any node of $C^{\prime}$,

- $s$ does not vanish identically on any irreducible component of $C^{\prime}$,

- the points $p_{1}, \ldots, p_{k}$ and $\operatorname{Div}(s)$ together stabilize $C^{\prime}$.

Good sections are open in the space of sections of $\left[C^{\prime}, L^{\prime}\right]$. The zeros of a good section define a twisted canonical divisor.

Since we started with a twisted canonical divisor, $\left[C, p_{1}, \ldots, p_{n}\right] \in \widetilde{\mathcal{H}}_{g}(\mu)$. We have a good section

$$
L\left(-\sum_{i=1}^{k} m_{i} p_{i}\right) \cong \mathcal{O}_{C}\left(\sum_{i=k+1}^{n} m_{i} p_{i}\right)
$$

associated to the pair $[C, L]$.

We can now estimate the dimension of the space of good sections of

$$
L^{\prime}\left(-\sum_{i=1}^{k} m_{i} p_{i}\right)
$$

as $\left[C^{\prime}, L^{\prime}\right]$ varies in $\mathcal{W}$ near $[C, L]$ using Proposition 14 of $\S 4.1$. The local dimension of the space of sections near $[C, L]$ is at least

$$
\begin{aligned}
\operatorname{dim} \mathcal{W}+\chi(C, L) & =3 g-3+k+2 r_{1}+r_{2}+g-1-\sum_{i=1}^{k} m_{i} \\
& =4 g-4+k+2 r_{1}+r_{2}-\sum_{i=1}^{k} m_{i}
\end{aligned}
$$

Hence, dimension of the space $\mathcal{T}$ of twisted canonical divisors on the fibers of

$$
\pi: \mathcal{C} \rightarrow \mathcal{W}
$$

near $\left[C, p_{1}, \ldots, p_{n}\right]$ is at least $4 g-5+k+2 r_{1}+r_{2}-\sum_{i=1}^{k} m_{i}$. 
We must further impose conditions on the twisted canonical divisors parameterized by $\mathcal{T}$ to obtain the shape $\sum_{i=k+1}^{n} m_{i} p_{i}$ for the positive part. These conditions impose at most

$$
2 g-2-\sum_{i=1}^{k} m_{i}-(n-k)=2 g-2+k-n-\sum_{i=1}^{k} m_{i}
$$

constraints. Hence, we conclude the dimension of space $\mathcal{T}(\mu)$ of twisted canonical divisors on the fibers of

$$
\pi: \mathcal{C} \rightarrow \mathcal{W}
$$

near $\left[C, p_{1}, \ldots, p_{n}\right]$ of shape $\mu$ is at least

$$
\begin{aligned}
& 4 g-5+k+2 r_{1}+r_{2}-\sum_{i=1}^{k} m_{i}-\left(2 g-2+k-n-\sum_{i=1}^{k} m_{i}\right) \\
& =2 g-3+n+2 r_{1}+r_{2} .
\end{aligned}
$$

Suppose $\left[C, p_{1}, \ldots, p_{n}\right] \in \widetilde{\mathcal{H}}_{g}(\mu)$ is a generic element of an irreducible component $Z \subset \widetilde{\mathcal{H}}_{g}(\mu)$ of dimension strictly less than $2 g-3+n$. In the versal deformation space $\mathcal{V}$ above, we consider the dimension of the sublocus

$$
\mathcal{Z} \subset \mathcal{T}(\mu)
$$

corresponding to twisted canonical divisors on the fibers of (19) which have the same dual graph as $C$. The dimension of the sublocus $\mathcal{Z}$ is equal to

$$
\operatorname{dim} Z+2 r_{1}+r_{2}
$$

which is less than $2 g-3+n+2 r_{1}+r_{2}$. The summands $2 r_{1}$ and $r_{2}$ appear here since we do not now quotient by the automorphism of the unstable components of the fibers.

We conclude at least one node of the curve $C$ can be smoothed in $\widetilde{\mathcal{H}}_{g}(\mu)$. Therefore, there does not exist a component $Z \subset \widetilde{\mathcal{H}}_{g}(\mu)$ of dimension strictly less than $2 g-3+n$.

\section{Theorems 2 and 3}

\subsection{Proof of Theorem 2}

By Proposition 4,

$$
\tilde{\mathcal{H}}_{g}(\mu) \subset \overline{\mathcal{M}}_{g, n}
$$

is a closed subvariety. In case all parts of $\mu=\left(m_{1}, \ldots, m_{n}\right)$ are nonnegative, every irreducible component of

$$
\mathcal{H}_{g}(\mu) \subset \mathcal{M}_{g, n}
$$

has dimension $2 g-2+n$. Hence, every irreducible component of

$$
\overline{\mathcal{H}}_{g}(\mu) \subset \overline{\mathcal{M}}_{g, n}
$$

has dimension $2 g-2+n$.

By Proposition 7 , every irreducible component of $\tilde{\mathcal{H}}_{g}(\mu)$ which is supported in the boundary $\partial \overline{\mathcal{M}}_{g, n}$ has dimension at most $2 g-3+n$. By Proposition 11, every irreducible component of $\widetilde{\mathcal{H}}_{g}(\mu)$ has dimension at least $2 g-3+n$. Hence, the boundary components of $\mathcal{H}_{g}(\mu)$ all have dimension $2 g-3+n$. 


\subsection{Locus of irreducible curves}

From the point of view of twists, the locus of stable pointed curves

$$
\mathcal{M}_{g, n}^{\mathrm{lrr}} \subset \overline{\mathcal{M}}_{g, n}
$$

with irreducible domains is very natural to consider,

$$
\left[C, p_{1}, \ldots, p_{n}\right] \in \mathcal{M}_{g, n}^{\mathrm{Irr}} \leftrightarrow C \text { is irreducible. }
$$

Since an irreducible curve has no basic nodes, a twisted canonical divisor

$$
\left[C, p_{1}, \ldots, p_{n}\right] \in \tilde{\mathcal{H}}_{g}(\mu) \cap \mathcal{M}_{g, n}^{\mathrm{lr}}
$$

is a usual canonical divisor

$$
\mathcal{O}_{C}\left(\sum_{i=1}^{n} m_{i} p_{i}\right) \stackrel{\sim}{=} \omega_{C} .
$$

We define $\mathcal{H}_{g}^{\mathrm{Irr}}(\mu)$ by the intersection

$$
\mathcal{H}_{g}^{\mathrm{Irr}}(\mu)=\widetilde{\mathcal{H}}_{g}(\mu) \cap \mathcal{M}_{g, n}^{\mathrm{Irr}}
$$

Lemma 12. If all parts of $\mu$ are nonnegative,

$$
\overline{\mathcal{H}}_{g}(\mu) \cap \mathcal{M}_{g, n}^{\mathrm{lrr}}=\mathcal{H}_{g}^{\mathrm{Irr}}(\mu) .
$$

Proof. We must prove there does not exist a component of $Z \subset \widetilde{\mathcal{H}}_{g}(\mu)$ generically supported in the boundary $\partial \mathcal{M}_{g, n}^{\mathrm{Ir}}$. We have seen that the dimension of $Z$ must be $2 g-3+n$. However, every irreducible component of the locus of canonical divisors in $\mathcal{M}_{g, n}^{\mathrm{Irr}}$ has dimension at least $2 g-2+n$ since the canonical bundle always has $g$ sections.

In the strictly meromorphic case, the equality

$$
\overline{\mathcal{H}}_{g}(\mu) \cap \mathcal{M}_{g, n}^{\mathrm{Irr}}=\mathcal{H}_{g}^{\mathrm{Irr}}(\mu) .
$$

also holds, but the result does not follow from elementary dimension arguments. Instead, the analytic perspective of flat surface is required, see [4, 26, 28].

By Lemma 12 in the holomorphic case and Theorem 3 in the strictly meromorphic case, we conclude the following two dimension results:

(i) If all parts of $\mu$ are nonnegative, $\mathcal{H}_{g}^{\mathrm{Irr}}(\mu)$ has pure dimension $2 g-2+n$.

(ii) If $\mu$ has a negative part, $\mathcal{H}_{g}^{\mathrm{Irr}}(\mu)$ has pure dimension $2 g-3+n$.

The dimensions (i) and (ii) are identical to the dimensions of $\mathcal{H}_{g}(\mu)$ in the corresponding cases.

Since $\overline{\mathcal{H}}_{g}(\mu)=\overline{\mathcal{H}}_{g}^{\mathrm{lrr}}(\mu)$ for all $\mu$, the star graphs of $\S 2.2$ with self-edges at the center vertex do not correspond to the dual graph $\Gamma_{Z}$ of a generic element of an irreducible component $Z \subset \widetilde{\mathcal{H}}_{g}(\mu)$ supported in the boundary $\partial \overline{\mathcal{M}}_{g, n}$. We state the result as a lemma. 
Lemma 13. The dual graph $\Gamma_{Z}$ of a generic element of an irreducible component

$$
Z \subset \tilde{\mathcal{H}}_{g}(\mu)
$$

supported in the boundary $\partial \overline{\mathcal{M}}_{g, n}$ is a star graph with no self-edges at the center.

Such star graphs will be called simple star graphs. In the Appendix A, in the strictly meromorphic case, we include in the set of simple star graphs the trivial star graph with a center vertex carrying all the parts of $\mu$ and no edges or outlying vertices.

\subsection{Proof of Theorem 3}

By Proposition 4,

$$
\tilde{\mathcal{H}}_{g}(\mu) \subset \overline{\mathcal{M}}_{g, n}
$$

is a closed subvariety. In case $\mu$ has at least one negative part, every irreducible component of

$$
\mathcal{H}_{g}(\mu) \subset \mathcal{M}_{g, n}
$$

has dimension $2 g-3+n$, and every irreducible component of $\tilde{\mathcal{H}}_{g}(\mu)$ which is supported in the boundary $\partial \overline{\mathcal{M}}_{g, n}$ has dimension at most $2 g-3+n$ by Proposition 7. By Proposition 11 every irreducible component of $\widetilde{\mathcal{H}}_{g}(\mu)$ has dimension at least $2 g-3+n$. Hence, $\widetilde{\mathcal{H}}_{g}(\mu)$ is of pure dimension $2 g-3+n$.

\section{Sections of line bundles}

\subsection{Dimension estimates}

Let $\mathcal{X}$ be a variety of pure dimension $d$, and let

$$
\pi: \mathcal{C} \rightarrow \mathcal{X}
$$

be a flat family of nodal curves. Let

$$
\mathcal{L} \rightarrow \mathcal{C}
$$

be a line bundle with Euler characteristic $\chi(\mathcal{L})$ on the fibers of $\pi$. Let

$$
\phi: \mathcal{M} \rightarrow \mathcal{X}
$$

be the variety parameterizing nontrivial sections of $\mathcal{L}$ on the fibers of $\pi$,

$$
\phi^{-1}(\xi)=H^{0}\left(\mathcal{C}_{\xi}, \mathcal{L}_{\xi}\right) \backslash\{0\}
$$

for $\xi \in \mathcal{X}$.

Proposition 14. Every irreducible component of $\mathcal{M}$ has dimension at least $d+\chi(\mathcal{L})$.

The result is immediate from the point of view of obstruction theory. For the convenience of the reader, we include an elementary proof based on the construction of the parameter space $\mathcal{M}$. 
Proof. Using a $\pi$-relatively ample bundle $\mathcal{N} \rightarrow \mathcal{C}$, we form a quotient

$$
\bigoplus_{i=1}^{r} \mathcal{N}^{-k_{i}} \rightarrow \mathcal{L} \rightarrow 0, \quad k_{i}>0
$$

Let $\mathcal{A}=\bigoplus_{i=1}^{r} \mathcal{N}^{-k_{i}}$ and consider the associated short exact sequence

$$
0 \rightarrow \mathcal{B} \rightarrow \mathcal{A} \rightarrow \mathcal{L} \rightarrow 0
$$

of bundles on $\mathcal{C}$. Since $\mathcal{A}$ is $\pi$-relatively negative, $\mathcal{A}$ and $\mathcal{B}$ have no sections on the fibers of $\pi$. Hence,

$$
V_{\mathcal{A}}=R^{1} \pi_{*}(\mathcal{A}) \quad \text { and } \quad V_{\mathcal{B}}=R^{1} \pi_{*}(\mathcal{B})
$$

are vector bundles of ranks $a$ and $b$ on $\mathcal{X}$. For $\xi \in \mathcal{X}$, we have

$$
0 \rightarrow H^{0}\left(\mathcal{C}_{\xi}, \mathcal{L}_{\xi}\right) \rightarrow V_{\mathcal{B}, \xi} \rightarrow V_{\mathcal{A}, \xi} \rightarrow H^{1}\left(\mathcal{C}_{\xi}, \mathcal{L}_{\xi}\right) \rightarrow 0 .
$$

Therefore, the ranks satisfy $b-a=\chi(L)$.

The sequence in cohomology associated to (20) yields a bundle map

$$
\gamma: V_{\mathcal{B}} \rightarrow V_{\mathcal{A}}
$$

on $\mathcal{X}$. Let $V_{\mathcal{B}}^{*}$ be the total space of $V_{\mathcal{B}}$ with the zero section removed,

$$
q: V_{\mathcal{B}}^{*} \rightarrow \mathcal{X} .
$$

The pull-back to $V_{\mathcal{B}}^{*}$ of $V_{\mathcal{B}}$ carries a tautological line bundle

$$
0 \rightarrow Q \rightarrow q^{*}\left(V_{\mathcal{B}}\right)
$$

By (21), the parameter space $\mathcal{M}$ sits in $V_{\mathcal{B}}^{*}$ as the zero locus of the canonical map

$$
Q \rightarrow q^{*}\left(V_{\mathcal{A}}\right)
$$

obtained from the composition

$$
Q \rightarrow q^{*}\left(V_{\mathcal{B}}\right) \stackrel{q^{*}(\gamma)}{\longrightarrow} q^{*}\left(V_{\mathcal{A}}\right)
$$

Since $\mathcal{X}$ is of pure dimension $d, V_{\mathcal{B}}^{*}$ is of pure dimension $d+b$. Finally, since the rank of $q^{*}\left(V_{\mathcal{A}}\right)$ is $a$, every irreducible component of $\mathcal{M}$ is of dimension at least

$$
d+b-a=d+\chi(\mathcal{L}),
$$

by standard dimension theory.

The following result is a consequence of the existence of a reduced obstruction theory (see the cosection method of [20]). Again, we give an elementary proof. An application will appear in $\S 4.2$ below.

Proposition 15. If there exists a trivial quotient on $\mathcal{X}$,

$$
R^{1} \pi_{*} \mathcal{L} \rightarrow \mathcal{O}_{\mathcal{X}} \rightarrow 0
$$

then every irreducible component of $\mathcal{M}$ has dimension at least $d+\chi(\mathcal{L})+1$. 
Proof. We must improve the dimension estimate in Proposition 14 by 1 . The long exact sequence obtained from (20) yields a quotient

$$
V_{\mathcal{A}} \rightarrow R^{1} \pi_{*}(L) \rightarrow 0
$$

on $\mathcal{X}$. Composing with the given quotient

$$
R^{1} \pi_{*}(\mathcal{L}) \rightarrow \mathcal{O}_{\mathcal{X}} \rightarrow 0
$$

yields a quotient

$$
V_{\mathcal{A}} \rightarrow \mathcal{O}_{\mathcal{X}} \rightarrow 0
$$

Let $K=\operatorname{Ker}\left(V_{\mathcal{A}} \rightarrow \mathcal{O}_{\mathcal{X}}\right)$. Then,

$$
K \rightarrow \mathcal{X}
$$

is a vector bundle of rank $a-1$. Since the image of

$$
\gamma: V_{\mathcal{B}} \rightarrow V_{\mathcal{A}}
$$

lies in $K, \mathcal{M}$ is in fact the zero locus of

$$
Q \rightarrow q^{*}(K)
$$

on $V_{\mathcal{B}}^{*}$. The dimension of every irreducible component of $\mathcal{M}$ is at least

$$
d+b-a+1=d+\chi(\mathcal{L})+1
$$

by dimension theory as before.

\subsection{Irreducible components in the boundary}

We consider here the holomorphic case: $\mu=\left(m_{1}, \ldots, m_{n}\right)$ is a vector of zero multiplicities satisfying

$$
m_{i} \geqslant 0, \quad \sum_{i=1}^{n} m_{i}=2 g-2 .
$$

Let $Z \subset \widetilde{\mathcal{H}}_{g}(\mu)$ be an irreducible component of dimension $2 g-3+n$ supported in the boundary $\partial \overline{\mathcal{M}}_{g, n}$, and let

$$
\left[C, p_{1}, \ldots, p_{n}\right] \in Z
$$

be a generic element with associated twist $I$. The dual graph $\Gamma_{C}$ of $C$ must be of the form described in $\S 2.1$ and Lemma $13: \Gamma_{C}$ is a simple star with a center vertex $v_{0}$, edges connecting $v_{0}$ to outlying vertices $v_{1}, \ldots, v_{k}$, and a distribution of the parts of $\mu$ (with all negative parts distributed to the center $v_{0}$ ).

By Lemma 12, there are no irreducible components $Z \subset \widetilde{\mathcal{H}}_{g}(\mu)$ supported in the boundary with generic dual graphs with no outlying vertices. All loci with such generic dual graphs are in the closure of $\mathcal{H}_{g}(\mu)$. A similar result holds in case there is a single outlying vertex.

Proposition 16. If all parts of $\mu$ are nonnegative, there are no irreducible components

$$
Z \subset \tilde{\mathcal{H}}_{g}(\mu)
$$

supported in the boundary with generic dual graph having exactly 1 outlying vertex. All such loci are in the closure of $\mathcal{H}_{g}(\mu)$. 
Proof. As in the proof of Proposition 11, we study the versal deformation space of $C$ where

$$
\left[C, p_{1}, \ldots, p_{n}\right] \in Z
$$

is a generic element. However, a more delicate argument is required here. We assume the generic twist $I$ is nontrivial. If the twist $I$ is trivial, then $\left[C, p_{1}, \ldots, p_{n}\right]$ is a usual canonical divisor and the proof of Proposition 11 directly applies. The nodes of $C$ corresponding to self-edges of $v_{0}$ cannot be twisted by $I$.

We assume ${ }^{14}$ the vertices $v_{0}$ and $v_{1}$ both have genus $g_{i}>0$. Let $\mathcal{V}$ be the versal deformation space of the unpointed nodal curve $C$,

$$
\operatorname{dim} \mathcal{V}=3 g-3
$$

Let $e_{1}$ be the number of edges connecting $v_{0}$ and $v_{1}$. The subvariety $\mathcal{S} \subset \mathcal{V}$ of curves preserving the nodes of $C$ corresponding to the edges connecting $v_{0}$ to $v_{1}$ is of codimension $e_{1}$.

We blow up $\mathcal{V}$ in the subvariety $\mathcal{S}$,

$$
v: \mathcal{V}_{1} \rightarrow \mathcal{V}
$$

The fiber of $\mathcal{V}_{1}$ above the original point $[C] \in \mathcal{V}$ is

$$
v^{-1}([C])=\mathbb{P}^{e_{1}-1} .
$$

Via pull-back, we have a universal family of curves

$$
\pi: \mathcal{C} \rightarrow \mathcal{V}_{1}
$$

The blow-up yields a nonsingular exceptional divisor

$$
E_{1} \subset \mathcal{V}_{1}
$$

which contains $v^{-1}([C])$. By locally base-changing $\mathcal{V}_{1}$ via the $r$ th-power of the equation of $E_{1}$, we can introduce transverse $A_{r-1}$-singularities along the nodes of the fibers of $\mathcal{C}$ corresponding to the edges $e_{1}$. The crepant resolution introduces a chain of $r-1$ rational curves.

The outcome after such a base change is a family

$$
\tilde{\pi}: \widetilde{C} \rightarrow \mathcal{V}_{1}
$$

with the following properties over the generic point $\xi \in v^{-1}([C])$ :

(i) $\widetilde{C}_{\xi}$ is a semistable curve obtained from $C$ by inserting chains of $r-1$ rational components at the nodes,

(ii) the total space of $\widetilde{C}$ is nonsingular near $\widetilde{C}_{\xi}$,

(iii) the components of $\widetilde{C}_{\xi}$ correspond to divisors in the total space of $\widetilde{C}$.

${ }^{14}$ In case $g=0$ vertices occur, we can rigidify with $q$ markings as in the proof of Proposition 11 . We leave the routine modification to the reader. 
After selecting $r$ appropriately, we can twist the total space $\widetilde{C}$ as specified by $I$. Such a geometric twisting is possible because of condition (iii) above.

We follow the construction of $\S 1.3$. There, a nonnegative twist $\gamma_{D}$ is associated to each component $D \subset \widetilde{C}_{\xi}$. More precisely, the construction assigns

$$
\gamma_{v_{0}}=0, \quad \gamma_{v_{1}}=\prod_{i=1}^{e_{1}} I\left(q_{i}, v_{0}\right)>0
$$

and strictly positive twists less than $\gamma_{v_{1}}$ for the additional rational components. By twisting by the divisor $-\gamma_{v_{1}} \cdot E_{1}$ near $\xi \in \mathcal{V}_{1}$, we can change the assignments to

$$
\widehat{\gamma}_{v_{0}}=-\prod_{i=1}^{e_{1}} I\left(q_{i}, v_{0}\right)<0, \quad \widehat{\gamma}_{v_{1}}=0
$$

and strictly negative twists greater than $\widehat{\gamma}_{v_{0}}$ for the additional rational components.

Using the twists (22), we obtain a line bundle

$$
\mathcal{L}=\omega_{\tilde{\pi}}\left(\sum_{D} \widehat{\gamma}_{D} \cdot[D]\right)
$$

where the sum is over the irreducible components of $\widetilde{C}_{\xi}$ for generic $\xi \in v^{-1}([C])$. The line bundle (23) is defined over an open set

$$
\mathcal{U} \subset \mathcal{V}_{1}
$$

which contains the generic element $\xi \in v^{-1}([C])$.

On the fibers of $\tilde{\pi}$ over $\mathcal{U}$,

$$
H^{1}\left(\omega_{\tilde{\pi}}\left(\sum_{D} \widehat{\gamma}_{D} \cdot[D]\right)\right) \stackrel{\sim}{=} H^{0}\left(\mathcal{O}\left(\sum_{D}-\widehat{\gamma}_{D} \cdot[D]\right)\right)^{\vee}
$$

by Serre duality. Since $-\widehat{\gamma}_{D} \geqslant 0$, we obtain a canonical section

$$
\mathbb{C} \rightarrow H^{0}\left(\mathcal{O}\left(\sum_{D}-\widehat{\gamma}_{D} \cdot[D]\right)\right)^{\vee}
$$

Since $-\widehat{\gamma}_{v_{1}}=0$, the canonical section does not vanish identically on any fiber of $\tilde{\pi}$ over $\mathcal{U}$. Hence, we obtain a canonical quotient

$$
H^{1}\left(\omega_{\tilde{\pi}}\left(\sum_{D} \widehat{\gamma}_{D} \cdot[D]\right)\right) \rightarrow \mathbb{C} \rightarrow 0
$$

We can now estimate the dimension of the space of good sections of $\mathcal{L}$ on the fibers of $\tilde{\pi}$ over $\mathcal{U}$ near the generic element of $v^{-1}([C])$ using Proposition 15 of $\S 4.1$. The dimension of the space of sections is at least

$$
\operatorname{dim} \mathcal{U}+\chi(C, L)+1=3 g-3+g-1+1=4 g-3 .
$$


Hence, dimension of the space $\mathcal{T}$ of twisted canonical divisors on the fibers of $\tilde{\pi}$ over $\mathcal{U}$ corresponding to twisted canonical line bundle $\mathcal{L}$ near the generic element of $v^{-1}([C])$ is at least $4 g-4$.

We must further impose conditions on the twisted canonical divisors parameterized by $\mathcal{T}$ to obtain the shape $\sum_{i=1}^{n} m_{i} p_{i}$. These conditions impose at most

$$
2 g-2-n
$$

constraints. Hence, we conclude the dimension of space $\mathcal{T}(\mu)$ of twisted canonical divisors on the fibers of

$$
\tilde{\pi}: \widetilde{\mathcal{C}} \rightarrow \mathcal{U}
$$

near $\left[C, p_{1}, \ldots, p_{n}\right]$ of shape $\mu$ is at least

$$
4 g-4-(2 g-2-n)=2 g-2+n .
$$

The result contradicts the dimension $2 g-3+n$ of $Z$.

By Lemma 12 and Proposition 16 in the holomorphic case, the loci

$$
Z \subset \tilde{\mathcal{H}}_{g}(\mu)
$$

corresponding to star graphs with 0 or 1 outlying vertices are contained in the closure of $\mathcal{H}_{g}(\mu)$. However, there are virtual components of $\widetilde{\mathcal{H}}_{g}(\mu)$ with more outlying vertices.

A simple example occurs in genus $g=2$ with $\mu=(1,1)$. A nonsingular pointed curve

$$
\left[C, p_{1}, p_{2}\right] \in \mathcal{M}_{2,2}
$$

lies in $\mathcal{H}_{2}(1,1)$ if and only if $\left\{p_{1}, p_{2}\right\} \subset C$ is a fiber of the unique degree 2 map

$$
C \rightarrow \mathbb{P}^{1}
$$

determined by the canonical series. An easy argument using admissible covers shows that the generic element of the virtual component corresponding to the star graph below is not in the closure of $\mathcal{H}_{2}(1,1)$.

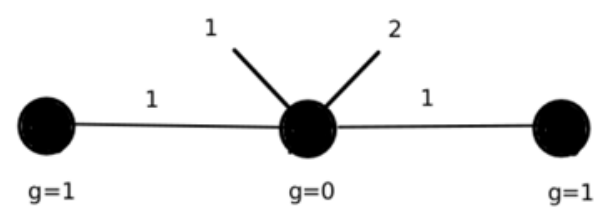

Both parts of $\mu$ are distributed to the center. The genera of the vertices and the twists of the edges are specified in the diagram. The above example (which occurs even in compact type) was discussed earlier by Chen [4].

\section{Twisted canonical divisors and limits of theta characteristics}

\subsection{Theta characteristics}

We illustrate the results of the paper (particularly Theorem 2 and Proposition 16) in two much studied cases linked to the classical theory of theta characteristics. Assume 
$\mu=\left(m_{1}, \ldots, m_{n}\right)$ consists of even nonnegative parts,

$$
m_{i}=2 a_{i}, \quad \sum_{i=1}^{n} a_{i}=g-1 .
$$

We write $\mu=2 \alpha$ where $\alpha \vdash g-1$.

If $\left[C, p_{1}, \ldots, p_{n}\right] \in \mathcal{H}_{g}(2 \alpha)$ is a canonical divisor, then

$$
\eta \stackrel{\sim}{=} \mathcal{O}_{C}\left(a_{1} p_{1}+\cdots+a_{n} p_{n}\right)
$$

is a theta characteristic. The study of the closure $\overline{\mathcal{H}}_{g}(2 \alpha)$ is therefore intimately related to the geometry of the moduli space $\overline{\mathcal{S}}_{g}$ of spin curves of genus $g$, see [7].

Two cases are of particular interest. If $\mu=(2 g-2)$ is of length one, $\mathcal{H}_{g}(2 g-2)$ is the locus of subcanonical pointed curves:

$$
[C, p] \in \mathcal{M}_{g, 1} \quad \Leftrightarrow \quad \omega_{C} \stackrel{\sim}{=} \mathcal{O}_{C}((2 g-2) p) .
$$

Subcanonical points are extremal Weierstrass points on the curve. For $g \geqslant 4$, Kontsevich and Zorich [21] have shown that the space $\mathcal{H}_{g}(2 g-2)$ has three connected components (of dimension $2 g-1$ ):

- the locus $\mathcal{H}_{g}(2 g-2)^{+}$of curves $[C, p]$ for which $\mathcal{O}_{C}((g-1) p)$ is an even theta characteristic,

- the locus $\mathcal{H}_{g}(2 g-2)^{-}$of curves $[C, p]$ for which $\mathcal{O}_{C}((g-1) p)$ is an odd theta characteristic,

- the locus $\mathcal{H}_{g}(2 g-2)^{\text {hyp }}$ of curves $[C, p]$ where $C$ is hyperelliptic and $p \in C$ is a Weierstrass point.

For $g=3$, the first and the third coincide

$$
\mathcal{H}_{3}(4)^{+}=\mathcal{H}_{3}(4)^{\text {hyp }},
$$

thus $\mathcal{H}_{3}(4)$ has only two connected components. For $g=2$, the space $\mathcal{H}_{2}(2)$ is irreducible. The geometry of the compactifications of these loci in small genera has been studied in [5].

The second case we consider is $\mu=(2, \ldots, 2)$ of length $g-1$. The space $\mathcal{H}_{g}(\underline{2})=$ $\mathcal{H}_{g}(2, \ldots, 2)$ splits into two connected components:

- the locus $\mathcal{H}_{g}(\underline{2})^{-}$of curves $\left[C, p_{1}, \ldots, p_{g-1}\right] \in \mathcal{M}_{g, g-1}$ for which

$$
\eta \stackrel{\sim}{=} \mathcal{O}_{C}\left(p_{1}+\cdots+p_{g-1}\right)
$$

is an odd theta characteristic,

- the locus $\mathcal{H}_{g}(\underline{2})^{+}$of curves $\left[C, p_{1}, \ldots, p_{g-1}\right] \in \mathcal{M}_{g, g-1}$ for which

$$
\eta \stackrel{\sim}{=} \mathcal{O}_{C}\left(p_{1}+\cdots+p_{g-1}\right)
$$

is a vanishing theta-null (even and $h^{0}(C, \eta) \geqslant 2$ ).

The component $\mathcal{H}_{g}(\underline{2})^{-}$is a generically finite cover of $\mathcal{M}_{g}$. For example, $\mathcal{H}_{3}(\underline{2})^{-}$is birationally isomorphic to the canonical double cover over the moduli space of bitangents of nonsingular quartic curves. On the other hand, the component $\mathcal{H}_{g}(\underline{2})^{+}$maps with 1 dimensional fibers over the divisor $\Theta_{\text {null }}$ of curves with a vanishing theta-null, see [12]. 


\subsection{Spin curves}

We require a few basic definitions concerning spin curves. A connected nodal curve $X$ is quasi-stable if, for every component

$$
E \stackrel{\sim}{=} \mathbb{P}^{1} \subset X
$$

the following two properties are satisfied:

- $k_{E}=|E \cap \overline{(X-E)}| \geqslant 2$,

- rational components $E, E^{\prime} \subset X$ with $k_{E}=k_{E^{\prime}}=2$ are always disjoint.

The irreducible components $E \subset X$ with $k_{E}=2$ are exceptional.

Definition 17 (Cornalba [7]). A stable spin curve of genus $g$ consists of a triple $(X, \eta, \beta)$ where:

(i) $X$ is a genus $g$ quasi-stable curve,

(ii) $\eta \in \operatorname{Pic}^{g-1}(X)$ is a line bundle of total degree $g-1$ with $\eta_{E}=\mathcal{O}_{E}(1)$ for all exceptional components $E \subset X$,

(iii) $\beta: \eta^{\otimes 2} \rightarrow \omega_{X}$ is a homomorphism of sheaves which is generically nonzero along each nonexceptional component of $X$.

If $(X, \eta, \beta)$ is a spin curve with exceptional components $E_{1}, \ldots, E_{r} \subset X$, then $\beta_{E_{i}}=0$ for $i=1, \ldots, r$ by degree considerations. Furthermore, if

$$
\tilde{X}=\overline{X-\bigcup_{i=1}^{r} E_{i}}
$$

is viewed as a subcurve of $X$, then we have an isomorphism

$$
\eta_{\widetilde{X}}^{\otimes 2} \stackrel{\sim}{\longrightarrow} \omega_{\widetilde{X}}
$$

The moduli space $\overline{\mathcal{S}}_{g}$ of stable spin curves of genus $g$ has been constructed by Cornalba [7]. There is a proper morphism

$$
\pi: \overline{\mathcal{S}}_{g} \rightarrow \overline{\mathcal{M}}_{g}
$$

associating to a spin curve $[X, \eta, \beta]$ the curve obtained from $X$ by contracting all exceptional components. The parity $h^{0}(X, \eta) \bmod 2$ of a spin curve is invariant under deformations. The moduli space $\overline{\mathcal{S}}_{g}$ splits into two connected components $\overline{\mathcal{S}}_{g}^{+}$and $\overline{\mathcal{S}}_{g}^{-}$ of relative degree $2^{g-1}\left(2^{g}+1\right)$ and $2^{g-1}\left(2^{g}-1\right)$ over $\overline{\mathcal{M}}_{g}$, respectively. The birational geometry of $\overline{\mathcal{S}}_{g}$ has been studied in $[12]$.

\subsection{Twisted canonical divisors on 2-component curves}

We describe the limits of supports of theta characteristics in case the underlying curve

$$
C=C_{1} \cup C_{2}
$$


is a union of two nonsingular curves $C_{1}$ and $C_{2}$ of genera $i$ and $g-i-\ell+1$ meeting transversally in a set of $\ell$ distinct points

$$
\Delta=\left\{x_{1}, \ldots, x_{\ell}\right\} \subset C .
$$

Let $2 a_{1} p_{1}+\cdots+2 a_{n} p_{n}$ be a twisted canonical divisor on $C$,

$$
\left[C, p_{1}, \ldots, p_{n}\right] \in \widetilde{\mathcal{H}}_{g}(2 \alpha)
$$

By definition, there exist twists

$$
I\left(x_{j}, C_{1}\right)=-I\left(x_{j}, C_{2}\right), \quad j=1, \ldots, \ell
$$

for which the following linear equivalences on $C_{1}$ and $C_{2}$ hold:

$\omega_{C_{1}} \equiv \sum_{p_{i} \in C_{1}} 2 a_{i} p_{i}-\sum_{j=1}^{\ell}\left(I\left(x_{j}, C_{1}\right)+1\right) x_{j} \quad$ and $\quad \omega_{C_{2}} \equiv \sum_{p_{i} \in C_{2}} 2 a_{i} p_{i}-\sum_{j=1}^{\ell}\left(I\left(x_{j}, C_{2}\right)+1\right) x_{j}$.

By Proposition 16, all twisted holomorphic differentials on $C$ are smoothable,

$$
\left[C, p_{1}, \ldots, p_{n}\right] \in \overline{\mathcal{H}}_{g}(\mu) .
$$

Following [7], we describe all spin structures having $C$ as underlying stable curve. If $[X, \eta, \beta] \in \pi^{-1}([C])$, then the number of nodes of $C$ where no exceptional component is inserted must be even. Hence, $X$ is obtained from $C$ by blowing up $\ell-2 h$ nodes. ${ }^{15}$ Let $\left\{x_{1}, \ldots, x_{2 h}\right\} \subset C$ be the non-blown-up nodes. We have

$$
X=C_{1} \cup C_{2} \cup E_{2 h+1} \cup \cdots \cup E_{\ell},
$$

where $C_{1} \cap E_{i}=\left\{x_{i}^{\prime}\right\}$ and $C_{2} \cap E_{i}=\left\{x_{i}^{\prime \prime}\right\}$ for $i=2 h+1, \ldots, \ell$ (see Figure 2). Furthermore,

$$
\eta_{E_{i}} \stackrel{\sim}{=} \mathcal{O}_{E_{i}}(1), \quad \eta_{C_{1}} \in \operatorname{Pic}^{i-1+h}\left(C_{1}\right), \quad \eta_{C_{2}} \in \operatorname{Pic}^{g-i-\ell+h}\left(C_{2}\right)
$$

with the latter satisfying

$$
\eta_{C_{1}}^{\otimes 2} \stackrel{\sim}{=} \omega_{C_{1}}\left(x_{1}+\cdots+x_{2 h}\right) \quad \text { and } \quad \eta_{C_{2}}^{\otimes 2} \stackrel{\sim}{=} \omega_{C_{2}}\left(x_{1}+\cdots+x_{2 h}\right) .
$$

There is a precise correspondence between the spin structures of Cornalba [7] and the system of twists associated to a point in $\widetilde{\mathcal{H}}_{g}(2 \alpha)$.

Proposition 18. Let $C=C_{1} \cup C_{2}$ as above. There exists a spin structure in $\overline{\mathcal{S}}_{g}$ corresponding to $\left[C, p_{1}, \ldots, p_{n}\right] \in \widetilde{\mathcal{H}}_{g}(2 \alpha)$ with twist $I$, which is obtained by blowing up $C$ at all the nodes $x_{j}$ where the twist

$$
I\left(x_{j}, C_{1}\right)=-I\left(x_{j}, C_{2}\right)
$$

is odd.

${ }^{15}$ The term blowing up here just means inserting an exceptional $E \stackrel{\sim}{=} \mathbb{P}^{1}$. 


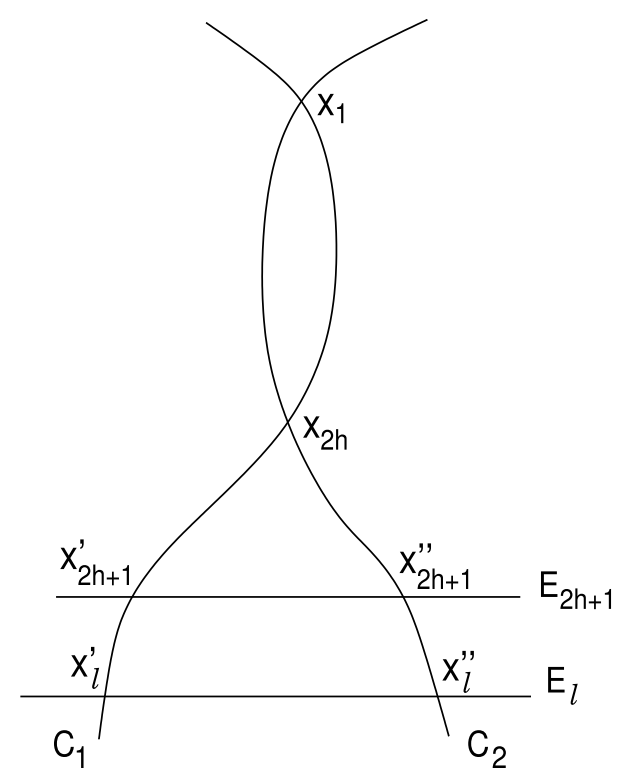

Figure 2. The quasi-stable curve $X$.

Proof. Since $\left[C, p_{1}, \ldots, p_{n}\right] \in \overline{\mathcal{H}}_{g}(2 \alpha)$ by Proposition 16 , there exists an $n$-pointed family of stable spin curves over a disc

$$
\mathcal{X} \rightarrow \Delta, \quad \eta \in \operatorname{Pic}(\mathcal{X}), \quad p_{1}, \ldots, p_{n}: \Delta \rightarrow \mathcal{X},
$$

satisfying:

- for $t \in \Delta^{\star}$, the fiber $X_{t}$ is nonsingular and

$$
\eta_{X_{t}} \stackrel{\sim}{=} \mathcal{O}_{X_{t}}\left(a_{1} p_{1}(t)+\cdots+a_{n} p_{n}(t)\right)
$$

- the central fiber $X_{0}$ is obtained from $C$ by inserting nonsingular rational components $E_{2 h+1}, \ldots, E_{\ell}$ at some of the nodes of $C$ which we assume to be $x_{2 h+1}, \ldots, x_{\ell}$,

- $\eta_{E_{j}} \stackrel{\sim}{=} \mathcal{O}_{E_{j}}(1)$ for $j=2 h+1, \ldots, \ell$.

By carrying out an argument following $\S 1.2$, after a finite base change and after resolution of singularities, we arrive at a new family

$$
\tilde{\pi}: \tilde{\mathcal{X}} \rightarrow \Delta
$$

with central fiber

$$
\mu: \tilde{\pi}^{-1}(0) \rightarrow X_{0}
$$

obtained from $X_{0}$ by inserting chains of nonsingular rational curves at the nodes of $X_{0}$ for which the line bundle

$$
\mu^{*}\left(\eta_{X}\right)\left(-a_{1} p_{1}-\cdots-a_{n} p_{n}\right)
$$

is the restriction to the central fiber of a line bundle on $\tilde{\mathcal{X}}$ supported only on the irreducible components of $\tilde{\pi}^{-1}(0)$. 
Just as in $\S 1.2$, we then obtain integral twists

$$
\tau_{j}=J\left(x_{j}, C_{1}\right)=-J\left(x_{j}, C_{2}\right)
$$

for $j=1, \ldots, 2 h$ and

$$
\tau_{j}^{\prime}=J\left(x_{j}^{\prime}, C_{1}\right)=-J\left(x_{j}^{\prime}, E_{j}\right) \quad \text { and } \quad \tau_{j}^{\prime \prime}=J\left(x_{j}^{\prime \prime}, E_{j}\right)=-J\left(x_{j}^{\prime \prime}, C_{2}\right)
$$

for $j=2 h+1, \ldots, \ell$, for which the restrictions of $\eta_{X}\left(-\sum_{i=1}^{n} a_{i} p_{i}\right)$ to the irreducible components of $X_{0}$ are isomorphic to the bundles given by the twists $J$ at the incident nodes. In particular,

$$
\eta_{E_{j}} \stackrel{\sim}{=} \mathcal{O}_{E_{j}}(1)=\mathcal{O}_{E_{j}}\left(-\tau_{j}^{\prime}+\tau_{j}^{\prime \prime}\right)
$$

hence $\tau_{j}^{\prime \prime}=\tau_{j}^{\prime}+1$ for $j=2 h+1, \ldots, \ell$. Furthermore, we have

$$
\begin{aligned}
\eta_{C_{1}}\left(-\sum_{p_{i} \in C_{1}} a_{i} p_{i}\right) & \stackrel{\sim}{=} \mathcal{O}_{C_{1}}\left(\tau_{1} x_{1}+\cdots+\tau_{2 h} x_{2 h}+\tau_{2 h+1}^{\prime} x_{2 h+1}^{\prime}+\cdots+\tau_{\ell}^{\prime} x_{\ell}^{\prime}\right), \\
\eta_{C_{2}}\left(-\sum_{p_{i} \in C_{2}} a_{i} p_{i}\right) & \stackrel{\sim}{=} \mathcal{O}_{C_{2}}\left(-\tau_{1} x_{1}-\cdots-\tau_{2 h} x_{2 h}-\tau_{2 h+1}^{\prime \prime} x_{2 h+1}^{\prime \prime}-\cdots-\tau_{\ell}^{\prime \prime} x_{\ell}^{\prime \prime}\right) .
\end{aligned}
$$

By squaring these relations and comparing to the original $I$ twists, we conclude

$$
I\left(x_{j}, C_{1}\right)=-2 \tau_{j}
$$

for $j=1, \ldots, 2 h$ and

$$
I\left(x_{j}, C_{1}\right)=-2 \tau_{j}^{\prime}-1
$$

for $j=2 h+1, \ldots, \ell$ respectively.

\subsection{Subcanonical points}

5.4.1. Genus 3. We illustrate Proposition 18 in case $^{16} g=3$ where

$$
\mathcal{H}_{3}(4)=\mathcal{H}_{3}(4)^{-} \sqcup \mathcal{H}_{3}(4)^{\text {hyp }} .
$$

Assume $C_{1}$ and $C_{2}$ are both nonsingular elliptic curves and $p \in C_{1} \backslash\left\{x_{1}, x_{2}\right\}$. Suppose

$$
\left[C_{1} \cup C_{2}, p\right] \in \overline{\mathcal{H}}_{3}(4) .
$$

The only possible twists are

$$
I\left(x_{1}, C_{1}\right)=I\left(x_{2}, C_{1}\right)=1 .
$$

The curve $\left[C_{1} \cup C_{2}, p\right] \in \overline{\mathcal{M}}_{3,1}$ lies in $\overline{\mathcal{H}}_{3}(4)$ if and only if the linear equivalence

$$
4 p \equiv 2 x_{1}+2 x_{2}
$$

holds on $C_{1}$.

${ }^{16}$ The case has also been treated by Chen in $[4, \S 7.2]$. 
The associated spin structure $[X, \eta]$ is obtained by inserting at both $x_{1}$ and $x_{2}$ rational components $E_{1}$ and $E_{2}$,

$$
X=C_{1} \cup C_{2} \cup E_{1} \cup E_{2}
$$

with the intersections

$$
C_{1} \cap E_{1}=\left\{x_{1}^{\prime}\right\}, \quad C_{1} \cap E_{2}=\left\{x_{2}^{\prime}\right\}, \quad C_{2} \cap E_{1}=\left\{x_{1}^{\prime \prime}\right\}, \quad C_{2} \cap E_{2}=\left\{x_{2}^{\prime \prime}\right\} .
$$

The line bundle $\eta$ satisfies

$$
\eta_{E_{i}} \stackrel{\sim}{=} \mathcal{O}_{E_{i}}(1), \quad \eta_{C_{1}} \stackrel{\sim}{=} \mathcal{O}_{C_{1}}\left(2 p-x_{1}-x_{2}\right), \quad \eta_{C_{2}} \stackrel{\sim}{=} \mathcal{O}_{C_{2}} .
$$

An argument via admissible covers implies

$$
[C, p] \in \overline{\mathcal{H}}_{3}(4)^{\text {hyp }} \quad \Leftrightarrow \quad \mathcal{O}_{C_{1}}\left(2 p-x_{1}-x_{2}\right) \stackrel{\sim}{=} \mathcal{O}_{C} .
$$

In the remaining case, where

$$
\eta_{C_{1}} \stackrel{\sim}{=} \mathcal{O}_{C_{1}}\left(2 p-x_{1}-x_{2}\right) \in J C_{1}[2]-\left\{\mathcal{O}_{C_{1}}\right\}
$$

is a nontrivial 2-torsion point, $\eta_{C_{1}}$ is an even and $\eta_{C_{2}}$ is an odd spin structure, so $[X, \eta] \in \overline{\mathcal{S}}_{3}^{-}$, and accordingly $[C, p] \in \overline{\mathcal{H}}_{3}(4)^{-}$.

5.4.2. Genus 4. Consider next genus 4 where

$$
\mathcal{H}_{4}(6)=\mathcal{H}_{4}(6)^{+} \sqcup \mathcal{H}_{4}(6)^{-} \sqcup \mathcal{H}_{4}(6)^{\text {hyp }}
$$

has three connected components of dimension 7. Let

$$
C=C_{1} \cup C_{2}, \quad g\left(C_{1}\right)=1, \quad g\left(C_{2}\right)=2
$$

with $C_{1} \cap C_{2}=\left\{x_{1}, x_{2}\right\}$. Suppose $p \in C_{1}$ and

$$
\left[C_{1} \cup C_{2}, p\right] \in \overline{\mathcal{H}}_{4}(6) \text {. }
$$

There are two possible twists.

- Twist $I\left(x_{1}, C_{1}\right)=I\left(x_{2}, C_{1}\right)=2$.

We then obtain the following linear equivalences on $C_{1}$ and $C_{2}$ :

$$
\mathcal{O}_{C_{1}}\left(6 p-3 x_{1}-3 x_{2}\right) \stackrel{\sim}{=} \mathcal{O}_{C_{1}} \text { and } \quad \omega_{C_{2}} \stackrel{\sim}{=} \mathcal{O}_{C_{2}}\left(x_{1}+x_{2}\right)
$$

Each equation appearing in (25) imposes a codimension 1 condition on the respective Jacobian. The corresponding spin curve in $[X, \eta] \in \overline{\mathcal{S}}_{4}$ has underlying model $X=C$ (no nodes are blown up), and the spin line bundle $\eta \in \operatorname{Pic}(X)$ satisfies

$$
\eta_{1} \stackrel{\sim}{=} \mathcal{O}_{C_{1}}\left(3 p-x_{1}-x_{2}\right) \text { and } \quad \eta_{2} \stackrel{\sim}{=} \mathcal{O}_{C_{2}}\left(x_{1}+x_{2}\right) .
$$

As expected, $\eta_{i}^{\otimes 2} \stackrel{\sim}{=} \omega_{C_{i}}\left(x_{1}+x_{2}\right)$ for $i=1,2$.

We can further distinguish between the components of the closure $\overline{\mathcal{H}}_{4}(6)$. We have $[C, p] \in \overline{\mathcal{H}}_{4}(6)^{\text {hyp }}$ if and only if

$$
\mathcal{O}_{C_{1}}\left(2 p-x_{1}-x_{2}\right) \stackrel{\sim}{=} \mathcal{O}_{C_{1}} \text { and } \quad \omega_{C_{2}} \stackrel{\sim}{=} \mathcal{O}_{C_{2}}\left(x_{1}+x_{2}\right),
$$


whereas $[C, p] \in \overline{\mathcal{H}}_{4}(6)^{-}$if and only if

$$
\mathcal{O}_{C_{1}}\left(2 p-x_{1}-x_{2}\right) \in J C_{1}[3]-\left\{\mathcal{O}_{C_{1}}\right\} \quad \text { and } \quad \omega_{C_{2}} \stackrel{\sim}{=} \mathcal{O}_{C_{2}}\left(x_{1}+x_{2}\right) \text {. }
$$

- Twist $I\left(x_{1}, C_{1}\right)=3$ and $I\left(x_{2}, C_{1}\right)=1$.

We then obtain the following linear equivalences on $C_{1}$ and $C_{2}$ :

$$
\mathcal{O}_{C_{1}}\left(6 p-4 x_{1}-2 x_{2}\right) \stackrel{\sim}{=} \mathcal{O}_{C_{1}} \text { and } \quad \omega_{C_{2}} \stackrel{\sim}{=} \mathcal{O}_{C_{2}}\left(2 x_{1}\right) .
$$

As before, the conditions in (26) are both codimension 1 in the respective Jacobians. The corresponding spin curve $[X, \eta]$ is obtained by blowing up both nodes $x_{1}$ and $x_{2}$,

$$
X=C_{1} \cup C_{2} \cup E_{1} \cup E_{2},
$$

with the intersections

$$
C_{1} \cap E_{1}=\left\{x_{1}^{\prime}\right\}, \quad C_{1} \cap E_{2}=\left\{x_{2}^{\prime}\right\}, \quad C_{2} \cap E_{1}=\left\{x_{1}^{\prime \prime}\right\}, \quad C_{2} \cap E_{2}=\left\{x_{2}^{\prime \prime}\right\} .
$$

The line bundle $\eta$ satisfies

$$
\eta_{E_{i}} \stackrel{\sim}{=} \mathcal{O}_{E_{i}}(1), \quad \eta_{C_{1}} \stackrel{\sim}{=} \mathcal{O}_{C_{1}}\left(3 p-2 x_{1}-x_{2}\right), \quad \eta_{C_{2}} \stackrel{\sim}{=} \mathcal{O}_{C_{2}}\left(x_{1}\right) .
$$

We have $[C, p] \in \overline{\mathcal{H}}_{4}(6)^{+}$if and only if $\eta_{C_{1}} \stackrel{\sim}{=} \mathcal{O}_{C_{1}}$. If $\eta_{C_{1}}$ is a nontrivial 2-torsion point, then $[C, p] \in \overline{\mathcal{H}}_{4}(6)^{-}$.

If $p \in C_{2}$ and $\left[C_{1} \cup C_{2}, p\right] \in \overline{\mathcal{H}}_{4}(6)$, then $I\left(x_{1}, C_{1}\right)=I\left(x_{2}, C_{1}\right)=-1$ is the only possible twist, and

$$
\omega_{C_{2}} \stackrel{\sim}{=} \mathcal{O}_{C_{2}}\left(6 p-2 x_{1}-2 x_{2}\right) .
$$

The corresponding spin structure is realized on the curve $X$ obtained by inserting rational components at both $x_{1}, x_{2} \in C$ and

$$
\eta_{C_{1}} \stackrel{\sim}{=} \mathcal{O}_{C_{1}}, \quad \eta_{C_{2}} \stackrel{\sim}{=} \mathcal{O}_{C_{2}}\left(3 p-x_{1}-x_{2}\right) .
$$

We have $[C, p] \in \overline{\mathcal{H}}_{4}(6)^{+}$(respectively $\overline{\mathcal{H}}_{4}(6)^{-}$) if and only if $\eta_{C_{2}}$ is an odd (respectively even) theta characteristic on $C_{2}$.

\subsection{Limits of bitangents in genus 3}

5.5.1. Two elliptic components. Recall the decomposition into components in the case of genus 3 :

$$
\mathcal{H}_{3}(2,2)=\mathcal{H}_{3}(2,2)^{-} \sqcup \mathcal{H}_{3}(2,2)^{+},
$$

where the general point $\left[C, p_{1}, p_{2}\right]$ of $\mathcal{H}_{3}(2,2)^{-}$corresponds to a bitangent line

$$
\left\langle p_{1}, p_{2}\right\rangle \in\left(\mathbb{P}^{2}\right)^{\vee}
$$

of the canonical model $C \subset \mathbb{P}^{2}$ and the general point of $\mathcal{H}_{3}(2,2)^{+}$corresponds to a hyperelliptic curve $C$ together with two points lying a fiber of the hyperelliptic pencil.

Let $C=C_{1} \cup C_{2}$ where $C_{1}$ and $C_{2}$ are nonsingular elliptic curves with

$$
C_{1} \cap C_{2}=\left\{x_{1}, x_{2}\right\} .
$$

Suppose $\left[C, p_{1}, p_{2}\right] \in \overline{\mathcal{H}}_{3}(2,2)$. There are two cases. 
Case 1: $p_{1}$ and $p_{2}$ lie on the same component of $C$, say $p_{1}, p_{2} \in C_{1} \backslash\left\{x_{1}, x_{2}\right\}$.

Then $I\left(x_{1}, C_{1}\right)=I\left(x_{2}, C_{1}\right)=1$, and the following equation holds:

$$
\mathcal{O}_{C_{1}}\left(2 x_{1}+2 x_{2}-2 p_{1}-2 p_{2}\right) \stackrel{\sim}{=} \mathcal{O}_{C_{1}} .
$$

The induced spin structure is obtained by blowing up both nodes $x_{1}$ and $x_{2}$, and the corresponding spin bundle $\eta$ restricts as

$$
\eta_{C_{1}} \stackrel{\sim}{=} \mathcal{O}_{C_{1}}\left(p_{1}+p_{2}-x_{1}-x_{2}\right) \quad \text { and } \quad \eta_{C_{2}} \stackrel{\sim}{=} \mathcal{O}_{C_{2}}
$$

If the linear equivalence $p_{1}+p_{2} \equiv x_{1}+x_{2}$ holds on $C_{1}$, then $\left[C, p_{1}, p_{2}\right] \in \overline{\mathcal{H}}_{3}(2,2)^{+}$as can be seen directly via admissible covers. ${ }^{17}$ If

$$
\eta_{C_{1}} \in J C_{1}[2]-\left\{\mathcal{O}_{C_{2}}\right\},
$$

then, since $\eta_{C_{2}}$ is odd, we have $\left[C, p_{1}, p_{2}\right] \in \overline{\mathcal{H}}_{3}(2,2)^{-}$. There is a 1-dimensional family of limiting bitangents to $C$, which is to be expected since $C$ is hyperelliptic.

Case 2: $p_{i} \in C_{i} \backslash\left\{x_{1}, x_{2}\right\}$ for $i=1,2$.

Then $I\left(x_{1}, C_{1}\right)=I\left(x_{2}, C_{1}\right)=0$. No node is twisted, so no rational components are inserted for the induced spin structure. The spin bundle $\eta \in \operatorname{Pic}^{2}(C)$ has restrictions

$$
\eta_{C_{i}} \stackrel{\sim}{=} \mathcal{O}_{C_{i}}\left(p_{i}\right)
$$

for $i=1,2$. Since the resulting spin curve is odd,

$$
\left[C, p_{1}, p_{2}\right] \in \overline{\mathcal{H}}_{3}(2,2)^{-} .
$$

We obtain $16=4 \times 4$ limits of bitangents corresponding to the choice of points $p_{i} \in C_{i}$ satisfying

$$
\mathcal{O}_{C_{i}}\left(2 p_{i}-x_{1}-x_{2}\right) \stackrel{\sim}{=} \mathcal{O}_{C_{i}} .
$$

We now study three further degenerate cases to offer an illustration of the transitivity condition in the definition of twists.

Case 3: $\left[C_{1} \cup C_{2} \cup E, p_{1}, p_{2}\right] \in \widetilde{\mathcal{H}}_{3}(2,2)$ with $p_{1} \in E \stackrel{\sim}{=} \mathbb{P}^{1}, p_{2} \in C_{1}$.

We have the following component intersections:

$$
C_{1} \cap C_{2}=\left\{x_{2}\right\}, \quad C_{1} \cap E=\left\{x_{1}^{\prime}\right\}, \quad C_{2} \cap E=\left\{x_{1}^{\prime \prime}\right\} .
$$

The twists are subject to three dependent equations. Let $a=I\left(x_{1}^{\prime}, C_{1}\right)$. We find

$$
I\left(x_{1}^{\prime \prime}, C_{2}\right)=-I\left(x_{1}^{\prime \prime}, E\right)=-2-a \quad \text { and } \quad I\left(x_{2}, C_{1}\right)=-I\left(x_{2}, C_{2}\right)=-a .
$$

If $a \leqslant-2$, the ordering on components defined by $I$,

$$
C_{1}<E \leqslant C_{2}<C_{1},
$$

${ }^{17}$ One can construct an admissible cover $f: C \rightarrow R$, where $R=\left(\mathbb{P}^{1}\right)_{1} \cup_{t}\left(\mathbb{P}^{1}\right)_{2}$, with restrictions $f_{\mid C_{i}}: C_{i} \rightarrow\left(\mathbb{P}^{1}\right)_{i}$ being the morphisms induced by the linear system $\left|\mathcal{O}_{C_{i}}\left(x_{1}+x_{2}\right)\right|$ for $i=1,2$ and $f^{-1}(t)=\left\{x_{1}, x_{2}\right\}$. Then clearly $f\left(p_{1}\right)=f\left(p_{2}\right)$. 
contradicts transitivity. Similarly, if $a \geqslant 0$, we obtain

$$
E \leqslant C_{1} \leqslant C_{2}<E
$$

which is also a contradiction. Therefore $a=-1$, and we have

$$
\mathcal{O}_{C_{1}}\left(2 p_{2}-2 x_{2}\right) \stackrel{\simeq}{=} \mathcal{O}_{C_{1}}
$$

The result is a 4-dimensional subvariety of $\overline{\mathcal{M}}_{3,2}$ and therefore cannot be a component of $\widetilde{\mathcal{H}}_{3}(2,2)$.

Case $4:\left[C_{1} \cup C_{2} \cup E_{1} \cup E_{2}, p_{1}, p_{2}\right] \in \tilde{\mathcal{H}}_{3}(2,2)$ with $p_{i} \in E_{i} \stackrel{\sim}{=} \mathbb{P}^{1}$.

We have the following component intersections:

$$
C_{1} \cap E_{1}=\left\{x_{1}^{\prime}\right\}, \quad C_{1} \cap E_{2}=\left\{x_{2}^{\prime}\right\}, \quad C_{2} \cap E_{1}=\left\{x_{1}^{\prime \prime}\right\}, \quad C_{2} \cap E_{2}=\left\{x_{2}^{\prime \prime}\right\} .
$$

Let $a=I\left(x_{1}^{\prime}, C_{1}\right)=-I\left(x_{1}^{\prime}, E_{1}\right)$. We obtain, after solving a system of equations,

$$
\begin{gathered}
I\left(x_{2}^{\prime}, C_{1}\right)=-I\left(x_{2}^{\prime}, E_{2}\right)=-2-a, \quad I\left(x_{2}^{\prime \prime}, E_{2}\right)=-I\left(x_{2}^{\prime \prime}, C_{2}\right)=-a, \\
I\left(x_{1}^{\prime \prime}, E_{1}\right)=-I\left(x_{1}^{\prime \prime}, C_{2}\right)=a+2 .
\end{gathered}
$$

When $a \leqslant-2$, we obtain

$$
C_{1}<E_{1} \leqslant C_{2} \leqslant E_{2} \leqslant C_{1},
$$

a contradiction. The case $a \geqslant 0$ is similarly ruled out, which forces $a=-1$. Then the twists impose no further constraints on the point in $\overline{\mathcal{M}}_{3,2}$, so we obtain again a 4-dimensional subvariety of $\widetilde{\mathcal{H}}_{3}(2,2)$ which cannot be an irreducible component.

Case 5: $\left[C_{1} \cup C_{2} \cup E, p_{1}, p_{2}\right] \in \widetilde{\mathcal{H}}_{3}(2,2)$ with $p_{1} \neq p_{2} \in E \stackrel{\sim}{=} \mathbb{P}^{1}$.

We have the following component intersections:

$$
C_{1} \cap C_{2}=\left\{x_{2}\right\}, \quad C_{1} \cap E=\left\{x_{1}^{\prime}\right\}, \quad C_{2} \cap E=\left\{x_{1}^{\prime \prime}\right\} .
$$

Arguments as above yield the conditions

$$
\mathcal{O}_{C_{1}}\left(2 x_{1}^{\prime}-2 x_{2}\right) \stackrel{\sim}{=} \mathcal{O}_{C_{1}} \quad \text { and } \quad \mathcal{O}_{C_{2}}\left(2 x_{1}^{\prime \prime}-2 x_{2}\right) \stackrel{\sim}{=} \mathcal{O}_{C_{2}}
$$

which constrains the locus to 3 dimensions in $\overline{\mathcal{M}}_{3,2}$.

5.5.2. Singular quartics. A nonsingular plane quartic curve

$$
C \subset \mathbb{P}^{2}
$$

is of genus 3 and has 28 bitangent lines corresponding to the set $\theta(C)$ of 28 odd theta characteristics. A general quartic $C \subset \mathbb{P}^{2}$ can be reconstructed from its 28 bitangent lines [3]: the rational map

$$
\overline{\mathcal{M}}_{3} \quad \rightarrow \quad \operatorname{Sym}^{28}\left(\mathbb{P}^{2}\right)^{\vee} / / \mathbb{P} \mathbb{G} \mathbb{L}(3), \quad C \mapsto \theta(C)
$$

is generically injective. Our approach to $\overline{\mathcal{H}}_{3}(2,2)$ naturally recovers classical results on the limits of bitangents for singular quartic curves. The investigation also further illustrates the difference between $\overline{\mathcal{H}}_{3}(2,2)$ and $\widetilde{\mathcal{H}}_{3}(2,2)$. 


\begin{tabular}{llll}
\hline$(\delta, \kappa)$ & $b_{0}$ & $b_{1}$ & $b_{2}$ \\
\hline$(0,0)$ & 28 & 0 & 0 \\
$(1,0)$ & 16 & 6 & 0 \\
$(2,0)$ & 8 & 8 & 1 \\
$(3,0)$ & 4 & 6 & 3 \\
$(0,1)$ & 10 & 6 & 0 \\
$(0,2)$ & 1 & 6 & 1 \\
$(0,3)$ & 1 & 0 & 3 \\
$(1,1)$ & 4 & 7 & 1 \\
\hline
\end{tabular}

TABLE 1. Bitangent data for singular quartic curves $[3,17]$.

Definition 19. Let $C \subset \mathbb{P}^{2}$ be an irreducible plane quartic. A line

$$
L \in\left(\mathbb{P}^{2}\right)^{\vee}
$$

is a bitangent of $C$ if the cycle $L \cdot C$ is everywhere nonreduced and even. A bitangent $L$ is of type $i$ if $L$ contains precisely $i$ singularities of $C$. For $i=0,1,2$, let $b_{i}$ denote the number of bitangent lines to $C$ of type $i$.

Let $C \subset \mathbb{P}^{2}$ be an irreducible plane quartic having $\delta$ nodes and $\kappa$ cusps. By Plücker's formulas involving the singularities of the dual plane curve, $C$ has

$$
b=b_{0}+b_{1}+b_{2}=28+2 \delta(\delta-7)+6 \kappa \delta+\frac{9 \kappa(\kappa-5)}{2}
$$

bitangent lines of which $b_{2}=\left(\begin{array}{c}\delta+\kappa \\ 2\end{array}\right)$ are of type 2 (see [3] and [17, Chapter XVII]). In Table 1 , the possibilities for $b_{i}$, depending on the singularities of $C$, are listed.

In order to recover the sum 28 in the each line of Table 1, multiplicities must be calculated. By [3, Lemma 3.3.1]:

- a bitangent of type 0 appears with multiplicity 1 in the scheme $\theta(C)$,

- a bitangent of type 1 containing a node (respectively a cusp) appears in $\theta(C)$ with multiplicity 2 (respectively 3),

- a bitangent joining two nodes (respectively two cusps) contributes to $\theta(C)$ with multiplicity 4 (respectively 9).

We explain how the results of Table 1 can be recovered using the geometry of $\widetilde{\mathcal{H}}_{3}(2,2)$ in two cases (the others are similar).

Case 1: 1-nodal quartics.

Let $\left[C, x_{1}, x_{2}\right] \in \mathcal{M}_{2,2}$ be a general point and denote by

$$
C^{\prime}=C / x_{1} \sim x_{2}
$$

the genus 3 curve obtained by identifying $x_{1}$ and $x_{2}$. If $\left[C^{\prime}, p_{1}, p_{2}\right] \in \widetilde{\mathcal{H}}_{3}(2,2)$, then

$$
\mathcal{O}_{C^{\prime}}\left(2 p_{1}+2 p_{2}\right) \stackrel{\sim}{=} \omega_{C^{\prime}}
$$


and $\mathcal{O}_{C}\left(2 p_{1}+2 p_{2}\right) \stackrel{\sim}{=} \omega_{C}\left(x_{1}+x_{2}\right)$. Since multiplication by 2 on $J C$ has degree 4 , there are 16 bitangents of type 0 . All 16 lie in $\overline{\mathcal{H}}_{3}(2,2)$ by Lemma 12.

We determine next the bitangents of type 1 . Let

$$
\left[C \cup E, p_{1}, p_{2}\right] \in \widetilde{\mathcal{H}}_{3}(2,2),
$$

where $E \stackrel{\sim}{=} \mathbb{P}^{1}, p_{1} \in E, p_{2} \in C$, and $E \cap C=\left\{x_{1}, x_{2}\right\}$. Then

$$
I\left(x_{1}, E\right)=I\left(x_{2}, E\right)=1
$$

and $\omega_{C}=\mathcal{O}_{C}\left(2 p_{2}\right)$. Hence, $p_{2}$ is one of the 6 Weierstrass points of $C$. Thus the bitangent lines of type 1 join a node and a Weierstrass point of the normalization. Each such bitangent line is counted with multiplicity 2 since $\left[C \cup E, p_{1}, p_{2}\right]$ has an automorphism of order 2. By Proposition 16, all 6 lie in $\overline{\mathcal{H}}_{3}(2,2)$.

Finally, we consider the case $\left[C \cup E, p_{1}, p_{2}\right] \in \widetilde{\mathcal{H}}_{3}(2,2)$ where

$$
p_{1}, p_{2} \in E-\left\{x_{1}, x_{2}\right\} .
$$

Then we find $I\left(x_{1}, C\right)+I\left(x_{2}, C\right)=4$. Both possibilities $\left\{I\left(x_{1}, C\right), I\left(x_{2}, C\right)\right\}=\{2,2\}$ and $\left\{I\left(x_{1}, C\right), I\left(x_{2}, C\right)\right\}=\{1,3\}$ impose a nontrivial condition on the curve $\left[C, x_{1}, x_{2}\right] \in \mathcal{M}_{2,2}$, so the case does not appear generically.

We have verified the line corresponding to $(1,0)$ in Table $1: b_{0}=16, b_{1}=6, b_{2}=0$, and $28=16+2 \cdot 6$.

Case 2: 1-cuspidal quartics.

We consider the compact type curve $C=C_{1} \cup C_{2}$, where $C_{i}$ are smooth curves of genus $i$ for $i=1,2$ and $C_{1} \cap C_{2}=\{x\}$. We furthermore assume $x \in C_{2}$ is not a Weierstrass point, so $C$ is not hyperelliptic. We determine the bitangents which have $C$ as the underlying curve. Suppose

$$
\left[C, p_{1}, p_{2}\right] \in \widetilde{\mathcal{H}}_{3}(2,2),
$$

so $p_{1}, p_{2} \in C \backslash\{x\}$. We distinguish several cases:

- $p_{1}, p_{2} \in C_{1} \Rightarrow I\left(x, C_{1}\right)=3$ and $\omega_{C_{2}}=\mathcal{O}_{C_{2}}(2 x)$. Since $x$ is not a Weierstrass point, the case is impossible.

- $p_{1} \in C_{1}$ and $p_{2} \in C_{2} \Rightarrow I\left(x, C_{1}\right)=1$. We obtain the conditions

$$
\mathcal{O}_{C_{1}}\left(2 p_{1}\right)=\mathcal{O}_{C_{1}}(2 x) \text { and } \omega_{C_{2}}=\mathcal{O}_{C_{2}}\left(2 p_{2}\right) \text {. }
$$

All solutions are smoothable by Proposition 16. The case accounts for 18 points in the fiber of the projection $\overline{\mathcal{H}}_{3}(2,2) \rightarrow \overline{\mathcal{M}}_{3}$ over $[C]$, and shows that the cuspidal curve

$$
\phi=\phi_{\omega_{C_{2}}(2 x)}: C_{2} \rightarrow \mathbb{P}^{2}
$$

has 6 bitangents of type 1 joining the cusp $\phi(x)$ with one of the Weierstrass points of $C$. Each such line is counted with multiplicity 3 corresponding to the nontrivial 2-torsion points of $\left[C_{1}, x\right] \in \overline{\mathcal{M}}_{1,1}$.

- $p_{1}, p_{2} \in C_{2} \Rightarrow I\left(x, C_{2}\right)=1$. We obtain the condition

$$
\mathcal{O}_{C_{2}}\left(2 p_{1}+2 p_{2}\right)=\omega_{C_{2}}(2 x) \text {. }
$$


The cohomology class of the images of both maps $f_{i}: C_{2} \rightarrow \operatorname{Pic}^{2}\left(C_{2}\right)$ given by

$$
f_{1}(y)=\mathcal{O}_{C_{2}}(2 y), \quad f_{2}(y)=\omega_{C_{2}}(2 x-2 y)
$$

equals $4 \theta \in H^{2}\left(\operatorname{Pic}^{2}\left(C_{2}\right), \mathbb{Z}\right)$, hence

$$
\left[f_{1}\left(C_{2}\right)\right] \cdot\left[f_{2}\left(C_{2}\right)\right]=16 \theta^{2}=32 .
$$

The cases where $p_{1}=x$ and $p_{2} \in C_{2}$ is a Weierstrass point must be discarded, so we are left with $10=16-6$ possibilities for the bitangent lines.

Suppose one of the points $p_{1}$ or $p_{2}$ tends to $x$. The case when both $p_{1}$ and $p_{2}$ merge to $x$ leads to $x \in C_{2}$ being a Weierstrass point and can be discarded. We are thus left with the following situation.

- $\left[C_{1} \cup E \cup C_{2}, p_{1}, p_{2}\right] \in \widetilde{\mathcal{H}}_{3}(2,2)$ with $p_{1} \in E \cong \mathbb{P}^{1}$ and $p_{2} \in C_{2}$. Let

$$
\{y\}=C_{1} \cap E \quad \text { and } \quad\{x\}=C_{2} \cap E .
$$

We compute $I\left(y, C_{1}\right)=-1$ and $I\left(x, C_{2}\right)=-1$; therefore, $x$ is a Weierstrass point of $C_{2}$.

The locus of such points forms a 5-dimensional component of $\widetilde{\mathcal{H}}_{3}(2,2)$ that lies entirely in the boundary $\partial \overline{\mathcal{M}}_{3,2}$ (as can be checked by residue restrictions or limit linear series arguments). The general point can not be smoothed.

We have verified the line corresponding to $(0,1)$ in Table $1: b_{0}=10, b_{1}=6, b_{2}=0$, and $28=10+3 \cdot 6$.

\section{Twisted $k$-canonical divisors}

\subsection{Moduli space}

Let $g$ and $n$ be in the stable range $2 g-2+n>0$, and let $k \in \mathbb{Z}_{\geqslant 0}$. Let

$$
\mu=\left(m_{1}, \ldots, m_{n}\right), \quad m_{i} \in \mathbb{Z}
$$

be a vector satisfying

$$
\sum_{i=1}^{n} m_{i}=k(2 g-2)
$$

We define the closed substack of $k$-canonical divisors $\mathcal{H}_{g}^{k}(\mu) \subset \mathcal{M}_{g, n}$ by

$$
\mathcal{H}_{g}^{k}(\mu)=\left\{\left[C, p_{1}, \ldots, p_{n}\right] \in \mathcal{M}_{g, n} \mid \mathcal{O}_{C}\left(\sum_{i=1}^{n} m_{i} p_{i}\right)=\omega_{C}^{k}\right\} .
$$

The main focus of the paper has been on differentials which is the $k=1$ case. Rational functions and quadratic differentials correspond to $k=0$ and $k=2$, respectively.

Definition 20. The divisor $\sum_{i=1}^{n} m_{i} p_{i}$ associated to $\left[C, p_{1}, \ldots, p_{n}\right] \in \overline{\mathcal{M}}_{g, n}$ is $k$-twisted canonical if there exists a twist $I$ for which

$$
v^{*} \mathcal{O}_{C}\left(\sum_{i=1}^{n} m_{i} p_{i}\right) \cong v^{*}\left(\omega_{C}^{k}\right) \otimes \mathcal{O}_{C_{I}}\left(\sum_{q \in \mathrm{N}_{I}} I\left(q, D_{q}^{\prime}\right) \cdot q^{\prime}+I\left(q, D_{q}^{\prime \prime}\right) \cdot q^{\prime \prime}\right)
$$

on the partial normalization $C_{I}$. 
We define the subset $\widetilde{\mathcal{H}}_{g}^{k}(\mu) \subset \overline{\mathcal{M}}_{g, n}$ parameterizing $k$-twisted canonical divisors by

$$
\tilde{\mathcal{H}}_{g}^{k}(\mu)=\left\{\left[C, p_{1}, \ldots, p_{n}\right] \in \overline{\mathcal{M}}_{g, n} \mid \sum_{i=1}^{n} m_{i} p_{i} \text { is a } k \text {-twisted canonical divisor }\right\} .
$$

By definition, we have

$$
\tilde{\mathcal{H}}_{g}^{k}(\mu) \cap \mathcal{M}_{g, n}=\mathcal{H}_{g}^{k}(\mu),
$$

so $\mathcal{H}_{g}^{k}(\mu) \subset \tilde{\mathcal{H}}_{g}^{k}(\mu)$ is an open set.

Theorem 21. The moduli space $\widetilde{\mathcal{H}}_{g}^{k}(\mu) \subset \overline{\mathcal{M}}_{g, n}$ is a closed substack with irreducible components all of dimension at least $2 g-3+n$.

Proof. We replace $\omega_{C}$ by $\omega_{C}^{k}$ in the proofs of Proposition 4, Lemmas 6 and 9, and Proposition 11. The tensor power $k$ plays no essential role in the arguments.

\subsection{Rational functions}

In the $k=0$ case of rational functions, the moduli space

$$
\widetilde{\mathcal{H}}_{g}^{0}(\mu) \subset \overline{\mathcal{M}}_{g, n}
$$

may have irreducible components of various dimensions. No result parallel to the simple dimension behavior of Theorems 2 and 3 holds.

Let $\mu=\left(m_{1}, \ldots, m_{n}\right)$ be a vector satisfying

$$
\sum_{i=1}^{n} m_{i}=0
$$

Let $\overline{\mathcal{M}}_{g}\left(\mathbb{P}^{1}, \mu\right)^{\sim}$ be the moduli space of stable maps to rubber $^{18}$ with ramification profiles over 0 and $\infty$ determined by the positive and negative part of $\mu$. The following $k=0$ result has a straightforward proof which we leave to the reader.

Proposition 22. The image of the canonical map $\overline{\mathcal{M}}_{g}\left(\mathbb{P}^{1}, \mu\right) \sim \overline{\mathcal{M}}_{g, n}$ equals

$$
\tilde{\mathcal{H}}_{g}^{0}(\mu) \subset \overline{\mathcal{M}}_{g, n}
$$

\subsection{Higher $k$}

For $k>1$, we do not know uniform dimension results for $\mathcal{H}_{g}^{k}(\mu)$. Is the locus

$$
\mathcal{H}_{g}^{k}(\mu) \subset \mathcal{M}_{g, n}
$$

pure of expected codimension $g$ ? Unfortunately, the answer is no: if all parts of $\mu$ are nonnegative and divisible by $k$, then the sublocus

$$
\mathcal{H}_{g}^{1}\left(\frac{1}{k} \cdot \mu\right) \subset \mathcal{H}_{g}^{k}(\mu)
$$

is of codimension $g-1$ in $\mathcal{M}_{g, n}$.

${ }^{18}$ See [11] for definitions and further references. 
Question. Perhaps construction (29) is the only source of impure dimension?

In the $k=2$ case of quadratic differentials, the dimension theory developed in $[22,27]$ answers the above question in the affirmative when

$$
m_{i} \in\{-1,0,1,2, \ldots\} \text { for all } 1 \leqslant i \leqslant n .
$$

An affirmative answer for all $g, \mu$, and $k>1$ would perhaps be expected.

Acknowledgements. We thank E. Clader, A. Eskin, C. Faber, J. Guéré, F. Janda, A. Pixton, A. Polishchuk, A. Sauvaget, R. Thomas, and D. Zvonkine for helpful discussions and correspondence concerning the moduli space of canonical divisors. At the Mathematische Arbeitstagung in Bonn in June 2015, there were several talks and hallway discussions concerning flat surfaces and the moduli of holomorphic differentials (involving D. Chen, S. Grushevsky, M. Möller, A. Zorich and others) which inspired us to write our perspective which had been circulating as notes for a few years. The precise connection to Pixton's cycle in the Appendix A was found in the summer of 2015.

G.F. was supported by the DFG Sonderforschungsbereich Raum-Zeit-Materie. R.P. was supported by the Swiss National Science Foundation and the European Research Council through grants SNF-200021-143274 and ERC-2012-AdG-320368-MCSK. R.P. was also supported by SwissMap and the Einstein Stiftung. We are particularly grateful to the Einstein Stiftung for supporting our collaboration in Berlin.

\section{Appendix A. The weighted fundamental class of $\tilde{\mathcal{H}}_{g}(\mu)$ by F. Janda, R. Pandharipande, A. Pixton, and D. Zvonkine}

\section{A.1. Stable graphs and strata}

In the strictly meromorphic case, the fundamental class of $\widetilde{\mathcal{H}}_{g}(\mu)$ weighted by intrinsic multiplicities is conjectured here to be an explicit cycle in the tautological ring $R^{*}\left(\overline{\mathcal{M}}_{g, n}\right)$ found by Pixton in 2014. The formula is written in term of a summation over stable graphs $\Gamma$ indexing the strata of $\overline{\mathcal{M}}_{g, n}$. The summand in Pixton's formula corresponding to $\Gamma$ is a product over vertex, marking, and edge factors. We review here the standard indexing of the strata of $\overline{\mathcal{M}}_{g, n}$ by stable graphs.

The strata of the moduli space of curves correspond to stable graphs

$$
\Gamma=\left(\mathrm{V}, \mathrm{H}, \mathrm{L}, \mathrm{g}: \mathrm{V} \rightarrow \mathbb{Z}_{\geqslant 0}, v: \mathrm{H} \rightarrow \mathrm{V}, \iota: \mathrm{H} \rightarrow \mathrm{H}\right)
$$

satisfying the following properties:

(i) $\mathrm{V}$ is a vertex set with a genus function $\mathrm{g}: \mathrm{V} \rightarrow \mathbb{Z}_{\geqslant 0}$;

(ii) $\mathrm{H}$ is a half-edge set equipped with a vertex assignment $v: \mathrm{H} \rightarrow \mathrm{V}$ and an involution $\iota$

(iii) E, the edge set, is defined by the 2-cycles of $\iota$ in $\mathrm{H}$ (self-edges at vertices are permitted);

(iv) L, the set of legs, is defined by the fixed points of $\iota$ and is endowed with a bijective correspondence with a set of markings; 
(v) the pair (V, E) defines a connected graph;

(vi) for each vertex $v$, the stability condition holds:

$$
2 \mathrm{~g}(v)-2+\mathrm{n}(v)>0,
$$

where $\mathrm{n}(v)$ is the valence of $\Gamma$ at $v$ including both half-edges and legs.

An automorphism of $\Gamma$ consists of automorphisms of the sets $\mathrm{V}$ and $\mathrm{H}$ which leave invariant the structures $\mathrm{L}, \mathrm{g}, v$, and $\iota$. Let $\operatorname{Aut}(\Gamma)$ denote the automorphism group of $\Gamma$.

The genus of a stable graph $\Gamma$ is defined by:

$$
\mathrm{g}(\Gamma)=\sum_{v \in V} \mathrm{~g}(v)+h^{1}(\Gamma) .
$$

A stratum of the moduli space $\overline{\mathcal{M}}_{g, n}$ of Deligne-Mumford stable curves naturally determines a stable graph of genus $g$ with $n$ legs by considering the dual graph of a generic pointed curve parameterized by the stratum. Let $\mathrm{G}_{g, n}$ be the set of isomorphism classes of stable graphs of genus $g$ with $n$ legs. The set $\mathrm{G}_{g, n}$ is finite.

To each stable graph $\Gamma$, we associate the moduli space

$$
\overline{\mathcal{M}}_{\Gamma}=\prod_{v \in \mathrm{V}} \overline{\mathcal{M}}_{\mathrm{g}(v), \mathrm{n}(v)} .
$$

Let $\pi_{v}$ denote the projection from $\overline{\mathcal{M}}_{\Gamma}$ to $\overline{\mathcal{M}}_{\mathrm{g}(v), \mathrm{n}(v)}$ associated to the vertex $v$. There is a canonical morphism

$$
\xi_{\Gamma}: \overline{\mathcal{M}}_{\Gamma} \rightarrow \overline{\mathcal{M}}_{g, n}
$$

with image $^{19}$ equal to the stratum associated to the graph $\Gamma$. To construct $\xi_{\Gamma}$, a family of stable pointed curves over $\overline{\mathcal{M}}_{\Gamma}$ is required. Such a family is easily defined by attaching the pull-backs of the universal families over each of the $\overline{\mathcal{M}}_{\mathrm{g}(v), \mathrm{n}(v)}$ along the sections corresponding to half-edges.

\section{A.2. Additive generators of the tautological ring}

Let $\Gamma$ be a stable graph. A basic class on $\overline{\mathcal{M}}_{\Gamma}$ is defined to be a product of monomials in $\kappa$ classes $^{20}$ at each vertex of the graph and powers of $\psi$ classes at each half-edge (including the legs),

$$
\gamma=\prod_{v \in \mathrm{V}} \prod_{i>0} \kappa_{i}[v]^{x_{i}[v]} \cdot \prod_{h \in \mathrm{H}} \psi_{h}^{y[h]} \in A^{*}\left(\overline{\mathcal{M}}_{\Gamma}\right),
$$

where $\kappa_{i}[v]$ is the $i$ th kappa class on $\overline{\mathcal{M}}_{\mathrm{g}(v), \mathrm{n}(v)}$. We impose the condition

$$
\sum_{i>0} i x_{i}[v]+\sum_{h \in \mathrm{H}[v]} y[h] \leqslant \operatorname{dim}_{\mathbb{C}} \overline{\mathcal{M}}_{\mathrm{g}(v), \mathrm{n}(v)}=3 \mathrm{~g}(v)-3+\mathrm{n}(v)
$$

19 The degree of $\xi_{\Gamma}$ is $|\operatorname{Aut}(\Gamma)|$.

${ }^{20}$ Our convention is $\kappa_{i}=\pi_{*}\left(\psi_{n+1}^{i+1}\right) \in A^{i}\left(\overline{\mathcal{M}}_{g, n}, \mathbb{Q}\right)$ where

$$
\pi: \overline{\mathcal{M}}_{g, n+1} \rightarrow \overline{\mathcal{M}}_{g, n}
$$

is the map forgetting the marking $n+1$. For a review of $\kappa$ and the cotangent $\psi$ classes, see [15]. 
at each vertex to avoid the trivial vanishing of $\gamma$. Here, $\mathrm{H}[v] \subset \mathrm{H}$ is the set of legs (including the half-edges) incident to $v$.

Consider the $\mathbb{Q}$-vector space $\mathcal{S}_{g, n}$ whose basis is given by the isomorphism classes of pairs $[\Gamma, \gamma]$, where $\Gamma$ is a stable graph of genus $g$ with $n$ legs and $\gamma$ is a basic class on $\overline{\mathcal{M}}_{\Gamma}$. Since there are only finitely many pairs $[\Gamma, \gamma]$ up to isomorphism, $\mathcal{S}_{g, n}$ is finite dimensional. The canonical map

$$
q: \mathcal{S}_{g, n} \rightarrow R^{*}\left(\overline{\mathcal{M}}_{g, n}\right)
$$

is surjective [15] and provides additive generators of the tautological ring. The kernel of $q$ is the ideal of tautological relations (see $[23, \S 0.3]$ ).

\section{A.3. Pixton's formula}

A.3.1. Weighting. Let $\mu=\left(m_{1}, \ldots, m_{n}\right)$ be a vector of zero and pole multiplicities satisfying

$$
\sum_{i=1}^{n} m_{i}=2 g-2 .
$$

It will be convenient to work with the shifted vector defined by

$$
\tilde{\mu}=\left(m_{1}+1, \ldots, m_{n}+1\right), \quad \tilde{m}_{i}=m_{i}+1 .
$$

Let $\Gamma$ be a stable graph of genus $g$ with $n$ legs. An admissible weighting of $\Gamma$ is a function on the set of half-edges,

$$
w: \mathrm{H}(\Gamma) \rightarrow \mathbb{Z},
$$

which satisfies the following three properties:

(i) $\forall h_{i} \in \mathrm{L}(\Gamma)$, corresponding to the marking $i \in\{1, \ldots, n\}$,

$$
w\left(h_{i}\right)=\tilde{m}_{i}
$$

(ii) $\forall e \in \mathrm{E}(\Gamma)$, corresponding to two half-edges $h(e), h^{\prime}(e) \in \mathrm{H}(\Gamma)$,

$$
w(h)+w\left(h^{\prime}\right)=0 ;
$$

(iii) $\forall v \in \mathrm{V}(\Gamma)$,

$$
\sum_{h \mapsto v} w(h)=2 \mathrm{~g}(v)-2+\mathrm{n}(v),
$$

where the sum is taken over all $\mathrm{n}(v)$ half-edges incident to $v$.

Let $r$ be a positive integer. An admissible weighting $\bmod r$ of $\Gamma$ is a function,

$$
w: \mathrm{H}(\Gamma) \rightarrow\{0, \ldots, r-1\},
$$

which satisfies exactly properties (i)-(iii) above, but with the equalities replaced, in each case, by the condition of congruence mod $r$. For example, for (i), we require

$$
w\left(h_{i}\right)=\tilde{m}_{i} \bmod r .
$$

Let $\mathrm{W}_{\Gamma, r}$ be the set of admissible weightings $\bmod r$ of $\Gamma$. The set $\mathrm{W}_{\Gamma, r}$ is finite. 
A.3.2. Pixton's cycle. Let $r$ be a positive integer. We denote by $\mathrm{P}_{g, \mu}^{d, r} \in R^{d}\left(\overline{\mathcal{M}}_{g, n}\right)$ the degree $d$ component of the tautological class

$$
\begin{aligned}
& \sum_{\Gamma \in \mathrm{G}_{g, n}} \sum_{w \in \mathrm{W}_{\Gamma, r}} \frac{1}{|\operatorname{Aut}(\Gamma)|} \frac{1}{r^{h^{1}(\Gamma)}} \xi_{\Gamma *}\left[\prod_{v \in \mathrm{V}(\Gamma)} \exp \left(-\kappa_{1}[v]\right) \prod_{i=1}^{n} \exp \left(\tilde{m}_{i}^{2} \psi_{h_{i}}\right)\right. \\
& \left.\quad \times \prod_{e=\left(h, h^{\prime}\right) \in \mathrm{E}(\Gamma)} \frac{1-\exp \left(-w(h) w\left(h^{\prime}\right)\left(\psi_{h}+\psi_{h^{\prime}}\right)\right)}{\psi_{h}+\psi_{h^{\prime}}}\right]
\end{aligned}
$$

The following fundamental polynomiality property of $\mathrm{P}_{g, \mu}^{d, r}$ has been proven by Pixton.

Proposition 23 (Pixton [24]). For fixed $g, \mu$, and $d$, the class

$$
\mathrm{P}_{g, \mu}^{d, r} \in R^{d}\left(\overline{\mathcal{M}}_{g, n}\right)
$$

is polynomial in $r$ for sufficiently large $r$.

We denote by $\mathrm{P}_{g, \mu}^{d}$ the value at $r=0$ of the polynomial associated to $\mathrm{P}_{g, \mu}^{d, r}$ by Proposition 6.3. In other words, $\mathrm{P}_{g, \mu}^{d}$ is the constant term of the associated polynomial in $r$. The cycle $\mathrm{P}_{g, \mu}^{d}$ was constructed by Pixton [24] in 2014.

If $d>g$, Pixton conjectured (and Clader and Janda have proven [6]) the vanishing

$$
\mathrm{P}_{g, \mu}^{d}=0 \in R^{*}\left(\overline{\mathcal{M}}_{g, n}\right) \text {. }
$$

If $d=g$, the class $\mathrm{P}_{g, \mu}^{g}$ is nontrivial. When restricted to the moduli of curves of compact type, $\mathrm{P}_{g, \mu}^{g}$ is related to canonical divisors via the Abel-Jacobi map by earlier work of Hain [16]. We propose a precise relationship between $\mathbf{P}_{g, \mu}^{g}$ and a weighted fundamental classes of the moduli space of twisted canonical divisors on $\overline{\mathcal{M}}_{g, n}$.

\section{A.4. The moduli of twisted canonical divisors}

We consider here the strictly meromorphic case where $\mu=\left(m_{1}, \ldots, m_{n}\right)$ has at least one negative part. We construct a cycle

$$
\mathrm{H}_{g, \mu} \in A^{g}\left(\overline{\mathcal{M}}_{g, n}\right)
$$

by summing over the irreducible components of the moduli space $\widetilde{\mathcal{H}}_{g}(\mu)$ of twisted canonical divisors defined in the paper.

Let $\mathrm{S}_{g, \mu} \subset \mathrm{G}_{g, n}$ be the set of simple star graphs defined by the following properties:

- $\Gamma \in \mathrm{S}_{g, \mu}$ has a single center vertex $v_{0}$;

- edges (possibly multiple) of $\Gamma$ connect $v_{0}$ to outlying vertices $v_{1}, \ldots, v_{k}$;

- the negative parts of $\mu$ are distributed to the center $v_{0}$ of $\Gamma$.

A simple star graph $\Gamma$ has no self-edges. Let $\mathrm{V}_{\text {out }}(\Gamma)$ denote the set of outlying vertices. The simplest star graph consists of the center alone with no outlying vertices.

A twist $I$ of a simple star graph is a function

$$
I: \mathrm{E}(\Gamma) \rightarrow \mathbb{Z}_{>0}
$$

satisfying: 
- for the center $v_{0}$ of $\Gamma$,

$$
2 \mathrm{~g}\left(v_{0}\right)-2+\sum_{e \mapsto v_{0}}(I(e)+1)=\sum_{i \mapsto v_{0}} m_{i}
$$

- for each outlying vertex $v_{j}$ of $\Gamma$,

$$
2 \mathrm{~g}\left(v_{j}\right)-2+\sum_{e \mapsto v_{j}}(-I(e)+1)=\sum_{i \mapsto v_{j}} m_{i} .
$$

Let $\operatorname{Tw}(\Gamma)$ denote the finite set of possible twists.

The notation $i \mapsto v$ in above equations denotes markings (corresponding to the parts of $\mu$ ) which are incident to the vertex $v$. We denote by $\mu[v]$ the vector consisting of the parts of $\mu$ incident to $v$. Similarly, $e \mapsto v$ denotes edges incident to the vertex $v$. We denote by $-I\left[v_{0}\right]-1$ the vector of values $-I(e)-1$ indexed by all edges incident to $v_{0}$, and by $I\left[v_{j}\right]-1$ the vector of values $I(e)-1$ indexed by all edges incident to an outlying vertex $v_{j}$.

We define the weighted fundamental class $\mathrm{H}_{g, \mu} \in A^{g}\left(\overline{\mathcal{M}}_{g, n}\right)$ of the moduli space $\widetilde{H}_{g}(\mu)$ of twisted canonical divisors in the strictly meromorphic case by

$$
\begin{aligned}
\mathrm{H}_{g, \mu}= & \sum_{\Gamma \in \mathrm{S}_{g, \mu}} \sum_{I \in \operatorname{Tw}(\Gamma)} \frac{\prod_{e \in \mathrm{E}(\Gamma)} I(e)}{|\operatorname{Aut}(\Gamma)|} \\
& \times \xi_{\Gamma *}\left[\left[\overline{\mathcal{H}}_{\mathrm{g}\left(v_{0}\right)}\left(\mu\left[v_{0}\right],-I\left[v_{0}\right]-1\right)\right] \prod_{v \in \mathrm{V}_{\text {out }}(\Gamma)}\left[\overline{\mathcal{H}}_{\mathrm{g}(v)}(\mu[v], I[v]-1)\right]\right] .
\end{aligned}
$$

The right side of the definition of $\mathrm{H}_{g, \mu}$ may be viewed as a sum over all irreducible components of

$$
Z \subset \widetilde{\mathcal{H}}_{g}(\mu)
$$

If the curves of $Z$ generically do not have a separating node,

$$
Z \subset \overline{\mathcal{H}}_{g}^{\mathrm{Irr}}(\mu) \subset \overline{\mathcal{M}}_{g, n},
$$

see $\S 3.2$. Since there is an equality of closures $\overline{\mathcal{H}}_{g}(\mu)=\overline{\mathcal{H}}_{g}^{\operatorname{Irr}}(\mu)$, we obtain

$$
Z \subset \overline{\mathcal{H}}_{g}(\mu) \subset \overline{\mathcal{M}}_{g, n}
$$

Hence, $Z$ contributes to the term corresponding to the trivial star graph

$$
\Gamma=\left\{v_{0}\right\}
$$

of genus $g$ carrying all the parts of $\mu$.

The trivial star graph $\Gamma$ has no edges, nothing to twist, and no automorphisms. Since $\xi_{\Gamma}$ is the identity map here, the term corresponding to $\Gamma$ is

$$
\left[\overline{\mathcal{H}}_{\mathrm{g}\left(v_{0}\right)}(\mu)\right] \in A^{\mathrm{g}\left(v_{0}\right)}\left(\overline{\mathcal{M}}_{\mathrm{g}\left(v_{0}\right), \mathrm{n}\left(v_{0}\right)}\right) .
$$


If $Z$ has a separating node, then, by $\S 2.2$ of the paper, $Z$ contributes to the term corresponding to the associated simple star graph. Every outlying vertex contributes the class

$$
\left[\overline{\mathcal{H}}_{\mathrm{g}\left(v_{j}\right)}\left(\mu\left[v_{j}\right], I\left[v_{j}\right]-1\right)\right] \in A^{\mathrm{g}\left(v_{j}\right)-1}\left(\overline{\mathcal{M}}_{\mathrm{g}\left(v_{j}\right), \mathrm{n}\left(v_{j}\right)}\right)
$$

corresponding to a canonical divisor in the holomorphic case (since the parts of $\mu\left[v_{j}\right]$ and $I\left[v_{j}\right]-1$ are nonnegative).

The formula for $\mathrm{H}_{g, \mu}$ differs from the usual fundamental class of $\widetilde{\mathcal{H}}_{g}(\mu)$ by the weighting factor $\prod_{e \in \mathrm{E}(\Gamma)} I(e)$ which is motivated by relative Gromov-Witten theory.

Conjecture A. In the strictly meromorphic case, $\mathrm{H}_{g, \mu}=2^{-g} \mathrm{P}_{g, \mu}^{g}$.

Our conjecture provides a completely geometric representative of Pixton's cycle in terms of twisted canonical divisors. The geometric situation here is parallel, but much better behaved, than the corresponding result of [18] proving Pixton's conjecture for the double ramification cycle (as the latter carries virtual contributions from contracted components of excess dimension).

Finally, we speculate that the study of volumes of the moduli spaces of meromorphic differentials may have a much simpler answer if summed over all the components of $\widetilde{\mathcal{H}}_{g}(\mu)$. How to properly define such volumes here is a question left for the future.

\section{A.5. Examples}

A.5.1. Genus at most 1. Conjecture A is trivial in case $g=0$ since both sides are the identity in $R^{0}\left(\overline{\mathcal{M}}_{0, n}\right)$. In case $g=1$, Conjecture $\mathrm{A}$ is nontrivial and true. The class $\mathrm{H}_{1, \mu}$ has contributions from boundary components. Our of proof Conjecture A in case $g=1$ is indirect. We prove:

- $\mathrm{H}_{1, \mu}$ equals the double ramification cycle in genus 1 associated to $\mu$ via geometric arguments.

- $2^{-1} \mathrm{P}_{1, \mu}^{1}$ equals Pixton's prediction for the double ramification cycle via direct analysis of the formulas.

Then, by the main result of [18], Conjecture A holds in $g=1$. An alternate approach via direct calculation is also available in $g=1$.

A.5.2. Genus 2. A more interesting example is $g=2$ with $\mu=(3,-1)$. We first enumerate all simple star graphs (together with their possible twists) which contribute to the cycle $\mathrm{H}_{2,(3,-1)}$ :

(i) $\Gamma=\left\{v_{0}\right\}, g\left(v_{0}\right)=2,|\operatorname{Aut}(\Gamma)|=1$.

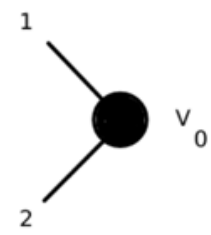


(ii) $\Gamma=\left\{v_{0}, v_{1}\right\}, \mathrm{g}\left(v_{0}\right)=1, \mathrm{~g}\left(v_{1}\right)=1,|\operatorname{Aut}(\Gamma)|=1$.

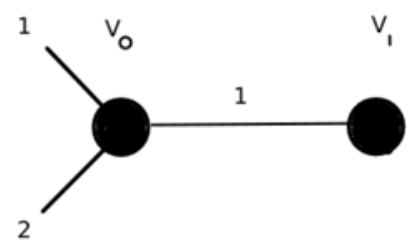

(iii) $\Gamma=\left\{v_{0}, v_{1}\right\}, \mathrm{g}\left(v_{0}\right)=0, \mathrm{~g}\left(v_{1}\right)=2,|\operatorname{Aut}(\Gamma)|=1$.

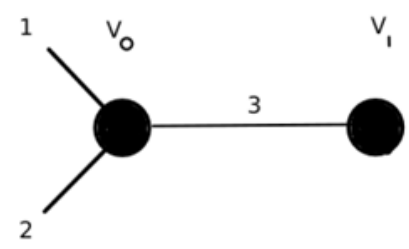

(iv) $\Gamma=\left\{v_{0}, v_{1}, v_{2}\right\}, \mathrm{g}\left(v_{0}\right)=0, \mathrm{~g}\left(v_{1}\right)=1, \mathrm{~g}\left(v_{2}\right)=1,|\operatorname{Aut}(\Gamma)|=2$.

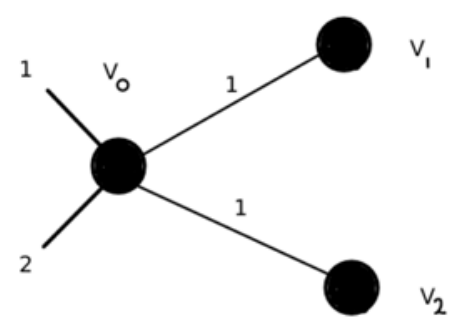

(v) $\Gamma=\left\{v_{0}, v_{1}\right\}, \mathrm{g}\left(v_{0}\right)=0, \mathrm{~g}\left(v_{1}\right)=1,|\operatorname{Aut}(\Gamma)|=2$.

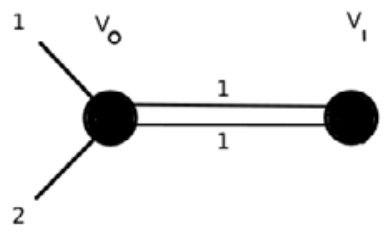

In the diagrams, the legs are assigned a marking, and the edges are assigned a twist. In all the cases $\Gamma$ above, the twist $I \in \operatorname{Tw}(\Gamma)$ is unique.

The second step is to calculate, for each star graph $\Gamma$ and associated twist $I \in \operatorname{Tw}(\Gamma)$, the contribution

$$
\frac{\prod_{e \in \mathrm{E}(\Gamma)} I(e)}{|\operatorname{Aut}(\Gamma)|} \xi_{\Gamma *}\left[\left[\overline{\mathcal{H}}_{\mathrm{g}\left(v_{0}\right)}\left(\mu\left[v_{0}\right],-I\left[v_{0}\right]-1\right)\right] \cdot \prod_{v \in \mathrm{V}_{\text {out }}(\Gamma)}\left[\overline{\mathcal{H}}_{\mathrm{g}(v)}(\mu[v], I[v]-1)\right]\right] .
$$

The contributions in cases (i)-(v) above are:

(i) The moduli space $\overline{\mathcal{H}}_{2}(3,-1)$ is empty since there are no meromorphic differentials with a simple pole. The contribution is 0 . 
(ii) We must calculate here $\overline{\mathcal{H}}_{1}(3,-1,-2)$ which is easily obtained from the method of test curves (or by applying Conjecture $\mathrm{A}$ in the proven genus 1 case). The contribution is
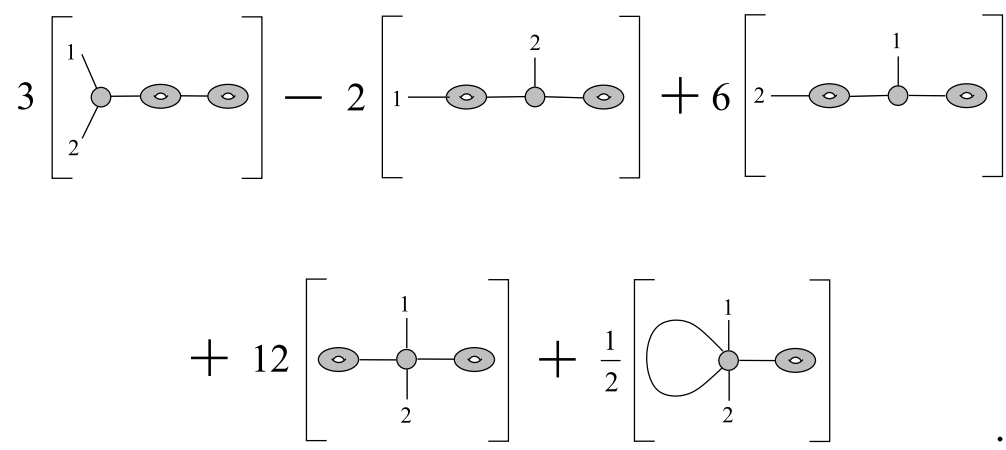

(iii) We require here the well-known formula for the Weierstrass locus,

$$
\overline{\mathcal{H}}_{2}(2) \subset \overline{\mathcal{M}}_{2,1},
$$

studied by Eisenbud and Harris [8]. Lemma 5 of [2] follows our notation here. The contribution, including the twist 3 , is

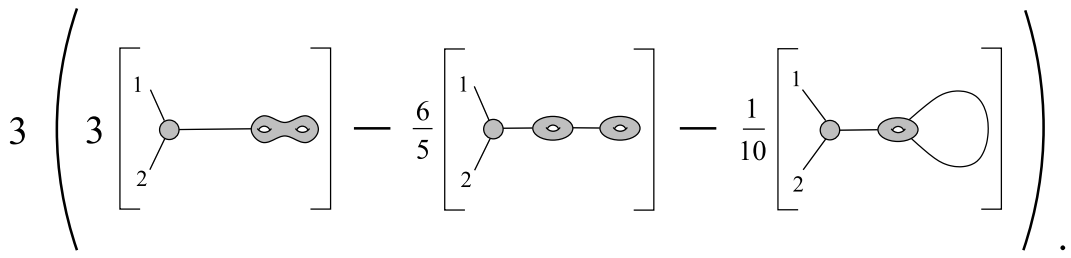

(iv) The locus is already codimension 2, so the contribution (including the automorphism factor) is

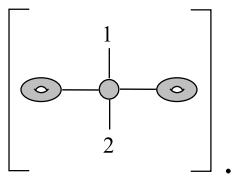

(v) The locus is again codimension 2, so the contribution (including the automorphism factor) is

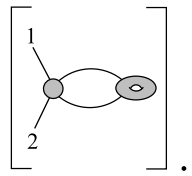

After summing over the cases (i)-(v), we obtain a formula in $R^{2}\left(\overline{\mathcal{M}}_{2,2}\right)$ for the weighted fundamental class $\mathrm{H}_{2,(3,-1)}$ of the moduli space of twisted canonical divisors: 

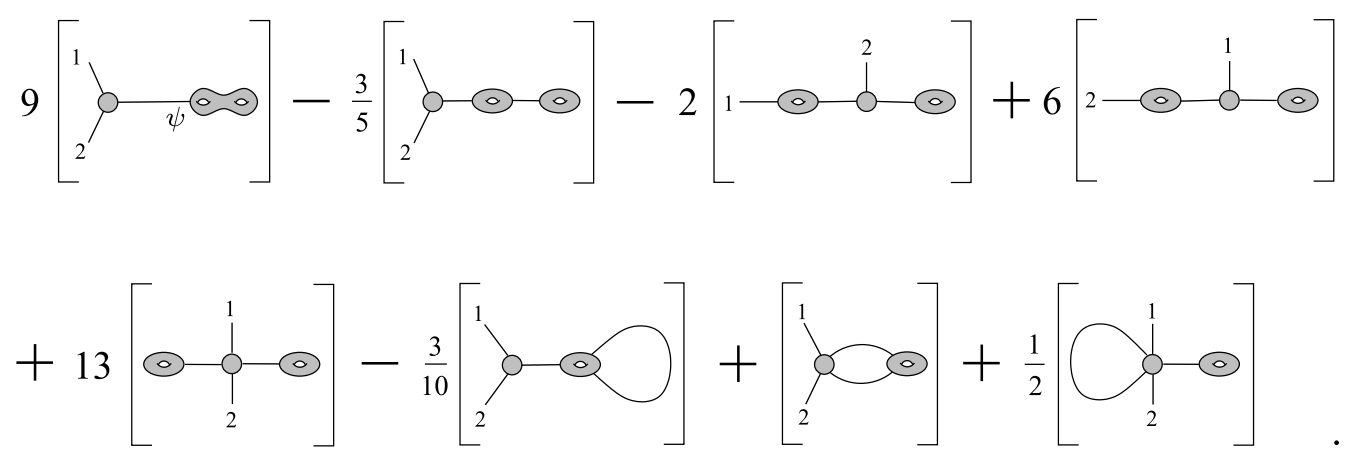

The result exactly equals $2^{-2} \mathrm{P}_{2,(3,-1)}^{2} \in R^{2}\left(\overline{\mathcal{M}}_{2,2}\right)$.

The match is obtained by expanding $2^{-2} \mathrm{P}_{2,(3,-1)}^{2}$ via the definition in $\S$ A.3.2 and then applying relations in the tautological ring. Conjecture A is therefore true for $g=2$ with $\mu=(3,-1)$.

We have also checked Conjecture A for $g=2$ with $\mu=(2,1,-1)$. The calculation of $\mathrm{H}_{2,(2,1,-1)}$ involves 7 simple star graphs, [2, Lemmas 5 and 6], and 23 strata classes of $R^{2}\left(\overline{\mathcal{M}}_{2,3}\right)$. The matching with

$$
2^{-2} \mathrm{P}_{2,(2,1,-1)}^{2} \in R^{2}\left(\overline{\mathcal{M}}_{2,3}\right)
$$

as predicted by Conjecture A exactly holds.

\section{A.6. The cycle $\overline{\mathcal{H}}_{g}(\mu)$}

A.6.1. Meromorphic differentials. In the strictly meromorphic case, where

$$
\mu=\left(m_{1}, \ldots, m_{n}\right)
$$

has at least one negative part, Conjecture A provides a formula in the tautological ring for the weighted fundamental class

$$
\mathrm{H}_{g, \mu} \in A^{g}\left(\overline{\mathcal{M}}_{g, n}\right) \text {. }
$$

Can Conjecture A also be used to determine the class

$$
\left[\overline{\mathcal{H}}_{g}(\mu)\right] \in A^{g}\left(\overline{\mathcal{M}}_{g, n}\right)
$$

of closure of the moduli of canonical divisors $\mathcal{H}_{g}(\mu)$ on nonsingular curves? We prove that the answer is yes.

We have seen $\left[\overline{\mathcal{H}}_{g}(\mu)\right]$ appears exactly as the contribution to $\mathrm{H}_{g, \mu}$ of the trivial simple star graph. Rewriting the definition of $\mathrm{H}_{g, \mu}$ yields the formula:

$$
\begin{aligned}
{\left[\overline{\mathcal{H}}_{g}(\mu)\right]=} & \mathrm{H}_{g, \mu}-\sum_{\Gamma \in \mathrm{S}_{g, \mu}^{\star}} \sum_{I \in \operatorname{Tw}(\Gamma)} \frac{\prod_{e \in \mathrm{E}(\Gamma)} I(e)}{|\operatorname{Aut}(\Gamma)|} \\
& \times \xi_{\Gamma *}\left[\left[\overline{\mathcal{H}}_{\mathrm{g}\left(v_{0}\right)}\left(\mu\left[v_{0}\right],-I\left[v_{0}\right]-1\right)\right] \prod_{v \in \mathrm{V}_{\text {out }}(\Gamma)}\left[\overline{\mathcal{H}}_{\mathrm{g}(v)}(\mu[v], I[v]-1)\right]\right]
\end{aligned}
$$

where $\mathrm{S}_{g, \mu}^{\star}$ is the set of nontrivial simple star graphs. 
A nontrivial simple star graph $\Gamma \in \mathrm{S}_{g, \mu}^{\star}$ must have at least one outlying vertex. An outlying vertex $v \in \mathrm{V}_{\text {out }}(\Gamma)$ contributes the factor

$$
\left[\overline{\mathcal{H}}_{\mathrm{g}(v)}(\mu[v], I[v]-1)\right]
$$

which concerns the holomorphic case. Hence if $\mathrm{g}(v)=0$, the contribution of $\Gamma$ vanishes (as there are no holomorphic differential in genus 0 ).

To state the first induction result, we require the standard partial order on the pairs $(g, n)$. We define

$$
(\widehat{g}, \widehat{n}) \stackrel{\circ}{<}(g, n)
$$

if $\widehat{g}<g$ holds or if

$$
\widehat{g}=g \text { and } \widehat{n}<n
$$

both hold.

Lemma A1. Let $\mu=\left(m_{1}, \ldots, m_{n}\right)$ be strictly meromorphic. The class

$$
\left[\overline{\mathcal{H}}_{g}(\mu)\right] \in A^{g}\left(\overline{\mathcal{M}}_{g, n}\right)
$$

is determined via formula (31) by classes of the following three types:

(i) $\mathrm{H}_{g, \mu} \in A^{g}\left(\overline{\mathcal{M}}_{g, n}\right)$;

(ii) $\left[\overline{\mathcal{H}}_{g^{\prime}}\left(\mu^{\prime}\right)\right] \in A^{g^{\prime}}\left(\overline{\mathcal{M}}_{g^{\prime}, n^{\prime}}\right) \quad$ for $\quad\left(g^{\prime}, n^{\prime}\right) \stackrel{\circ}{<}(g, n) \quad$ and $\quad \mu^{\prime}=\left(m_{1}^{\prime}, \ldots, m_{n^{\prime}}^{\prime}\right) \quad$ strictly meromorphic;

(iii) $\left[\overline{\mathcal{H}}_{g^{\prime \prime}}\left(\mu^{\prime \prime}\right)\right] \in A^{g^{\prime \prime}-1}\left(\overline{\mathcal{M}}_{g^{\prime \prime}, n^{\prime \prime}}\right) \quad$ for $\quad\left(g^{\prime \prime}, n^{\prime \prime}\right) \stackrel{\circ}{<}(g, n) \quad$ and $\quad \mu^{\prime \prime}=\left(m_{1}^{\prime \prime}, \ldots, m_{n^{\prime \prime}}^{\prime \prime}\right)$ holomorphic.

Proof. The class (i) appears as the leading term on the right side of (31). Nontrivial simple star graphs $\Gamma$ contribute classes of type (ii) via the center vertex $v_{0}$ (with

$$
g^{\prime}=\mathrm{g}\left(v_{0}\right)<g
$$

or else the contribution of $\Gamma$ vanishes). The outlying vertices $v$ of $\Gamma$ contribute classes of type (iii). If

$$
g^{\prime \prime}=\mathrm{g}(v)=g,
$$

then $\mathrm{g}\left(v_{0}\right)=0$ and there are no outlying vertices other than $v$ (or else the contribution of $\Gamma$ vanishes) and only a single edge. By stability, at least two parts of $\mu$ must be distributed to $v_{0}$, so $n^{\prime \prime}<n$.

Lemma A1 alone does not let us recursively calculate $\left[\overline{\mathcal{H}}_{g}(\mu)\right]$ in terms of $\mathrm{H}_{g, \mu}$ because of occurrences of the holomorphic cases (iii).

A.6.2. Holomorphic differentials. Consider the holomorphic case where

$$
\mu=\left(m_{1}, \ldots, m_{n}\right)
$$

has only nonnegative parts. Since

$$
\sum_{i=1}^{n} m_{i}=2 g-2
$$


we must have $g \geqslant 1$. If $g=1$, then all parts of $\mu$ must be 0 and

$$
\left[\overline{\mathcal{H}}_{1}(0, \ldots, 0)\right]=1 \in A^{0}\left(\overline{\mathcal{M}}_{1, n}\right) .
$$

We assume $g \geqslant 2$.

Let $\mu^{\prime}=\left(m_{1}^{\prime}, \ldots, m_{n^{\prime}}^{\prime}\right)$ be obtained from $\mu$ by removing the parts equal to 0 . Then $\left[\overline{\mathcal{H}}_{g}(\mu)\right]$ is obtained by pull-back ${ }^{21}$ via the map

$$
\tau: \overline{\mathcal{M}}_{g, n} \rightarrow \overline{\mathcal{M}}_{g, n^{\prime}}
$$

forgetting the markings associated to the 0 parts of $\mu$,

$$
\left[\overline{\mathcal{H}}_{g}(\mu)\right]=\tau^{*}\left[\overline{\mathcal{H}}_{g}\left(\mu^{\prime}\right)\right] \in A^{g-1}\left(\overline{\mathcal{M}}_{g, n}\right) .
$$

We assume $\mu$ has no 0 parts, so all parts $m_{i}$ are positive. We place the parts of $\mu$ in increasing order

$$
m_{1} \leqslant m_{2} \leqslant \cdots \leqslant m_{n-1} \leqslant m_{n},
$$

so $m_{n}$ is the largest part. Let $\mu^{+}$be the partition defined by

$$
\mu^{+}=\left(m_{1}, \ldots, m_{n-1}, m_{n}+1,-1\right) .
$$

We have increased the largest part of $\mu$, added a negative part, and preserved the sum

$$
|\mu|=\left|\mu^{+}\right| \text {. }
$$

For notational convenience, we write

$$
\mu^{+}=\left(m_{1}^{+}, \ldots, m_{n}^{+},-1\right), \quad m_{i<n}^{+}=m_{i}, \quad m_{n}^{+}=m_{n}+1 .
$$

The length of $\mu^{+}$is $n+1$.

Since $\mu^{+}$is strictly meromorphic, we are permitted to apply formula (31). We obtain

$$
\begin{aligned}
{\left[\overline{\mathcal{H}}_{g}\left(\mu^{+}\right)\right]=} & \mathrm{H}_{g, \mu^{+}}-\sum_{\Gamma \in \mathrm{S}_{g, \mu^{+}}^{\star}} \sum_{I \in \operatorname{Tw}(\Gamma)} \frac{\prod_{e \in \mathrm{E}(\Gamma)} I(e)}{|\operatorname{Aut}(\Gamma)|} \\
& \times \xi_{\Gamma *}\left[\left[\overline{\mathcal{H}}_{\mathrm{g}\left(v_{0}\right)}\left(\mu^{+}\left[v_{0}\right],-I\left[v_{0}\right]-1\right)\right] \cdot \prod_{v \in \mathrm{V}_{\text {out }}(\Gamma)}\left[\overline{\mathcal{H}}_{\mathrm{g}(v)}\left(\mu^{+}[v], I[v]-1\right)\right]\right]
\end{aligned}
$$

where $\mathrm{S}_{g, \mu^{+}}^{\star}$ is the set of nontrivial simple star graphs.

Since a meromorphic differential on a nonsingular curve can not have just one simple pole, $\mathcal{H}_{g}\left(\mu^{+}\right)$is empty and $\left[\overline{\mathcal{H}}_{g}\left(\mu^{+}\right)\right]=0$. We rewrite the above equation as

$$
\begin{aligned}
\mathrm{H}_{g, \mu^{+}}= & \sum_{\Gamma \in \mathrm{S}_{g, \mu^{+}}^{\star}} \sum_{I \in \operatorname{Tw}(\Gamma)} \frac{\prod_{e \in \mathrm{E}(\Gamma)} I(e)}{|\operatorname{Aut}(\Gamma)|} \\
& \times \xi_{\Gamma *}\left[\left[\overline{\mathcal{H}}_{\mathrm{g}\left(v_{0}\right)}\left(\mu^{+}\left[v_{0}\right],-I\left[v_{0}\right]-1\right)\right] \prod_{v \in \mathrm{V}_{\text {out }}(\Gamma)}\left[\overline{\mathcal{H}}_{\mathrm{g}(v)}\left(\mu^{+}[v], I[v]-1\right)\right]\right] .
\end{aligned}
$$

${ }^{21}$ The pull-back is true on the level of subvarieties, $\overline{\mathcal{H}}_{g}(\mu)=\tau^{-1}\left(\overline{\mathcal{H}}_{g}\left(\mu^{\prime}\right)\right) \subset \overline{\mathcal{M}}_{g, n}$. 
A nonvanishing term on right side of $(34)$ corresponding to $\Gamma \in \mathrm{S}_{g, \mu^{+}}^{\star}$ has a center vertex factor

$$
\left[\overline{\mathcal{H}}_{\mathrm{g}\left(v_{0}\right)}\left(\mu^{+}\left[v_{0}\right],-I\left[v_{0}\right]-1\right)\right] \text { satisfying } \mathrm{g}\left(v_{0}\right)<g
$$

and outlying vertex factors

$$
\left[\overline{\mathcal{H}}_{\mathrm{g}(v)}\left(\mu^{+}[v], I[v]-1\right)\right]
$$

satisfying either $\mathrm{g}(v)<g$ or $\mathrm{g}(v)=g$. If $\mathrm{g}(v)=g$, then the entire genus of $\Gamma$ is concentrated on $v$ and there can be no other outlying vertices (or else the contribution of $\Gamma$ vanishes) and only a single edge. By stability, at least two parts of $\mu^{+}$must be distributed to $v_{0}$, so $\mathrm{n}(v) \leqslant n$. Hence, the outlying vertex factors (35) satisfy either $\mathrm{g}(v)<g$ or

$$
\mathrm{g}(v)=g \quad \text { and } \quad \mathrm{n}(v) \leqslant n .
$$

We study now all the contributions of graphs $\Gamma \in \mathrm{S}_{g, \mu^{+}}^{\star}$ which carry an outlying vertex $v \in \mathrm{V}_{\text {out }}(\Gamma)$ satisfying

$$
\mathrm{g}(v)=g \quad \text { and } \quad \mathrm{n}(v)=n,
$$

the extremal case of $(36)$. We have seen

$$
\Gamma=\left\{v_{0}, v_{1}\right\} \text { with a single edge } e \text { and } \mathrm{g}\left(v_{0}\right)=0, \mathrm{~g}\left(v_{1}\right)=g
$$

is the only possibility for a nonvanishing contribution. By definition, the negative part of $\mu^{+}$must be distributed to $v_{0}$. In order for $\mathrm{n}\left(v_{1}\right)=n$, exactly one positive part $m_{i}^{+}$of $\mu^{+}$must also be distributed to $v_{0}$. Let $\Gamma_{i} \in \mathrm{S}_{g, \mu^{+}}^{\star}$ be the simple star graph of type (37) with marking distribution

$$
\left\{m_{i}^{+},-1\right\} \mapsto v_{0}, \quad\left\{m_{1}^{+}, \ldots, \widehat{m_{i}^{+}}, \ldots, m_{n}^{+}\right\} \mapsto v_{1} .
$$

The graphs $\Gamma_{1}, \Gamma_{2}, \ldots, \Gamma_{n}$ are the only elements of $\mathrm{S}_{g, \mu^{+}}^{\star}$ which saturate the bounds (36) and have possibly nonvanishing contributions to the right side of (34).

The negative part of $\mu^{+}$corresponds to the last marking of the associated moduli space $\overline{\mathcal{M}}_{g, n+1}$. Let

$$
\epsilon: \overline{\mathcal{M}}_{g, n+1} \rightarrow \overline{\mathcal{M}}_{g, n}
$$

be the map forgetting the last marking. We push forward formula (34) under $\epsilon$,

$$
\begin{aligned}
\epsilon_{*} \mathrm{H}_{g, \mu^{+}}= & \sum_{\Gamma \in \mathrm{S}_{g, \mu^{+}}^{\star}} \sum_{I \in \operatorname{Tw}(\Gamma)} \frac{\prod_{e \in \mathrm{E}(\Gamma)} I(e)}{|\operatorname{Aut}(\Gamma)|} \\
& \times \epsilon_{*} \xi_{\Gamma *}\left[\left[\overline{\mathcal{H}}_{\mathrm{g}\left(v_{0}\right)}\left(\mu^{+}\left[v_{0}\right],-I\left[v_{0}\right]-1\right)\right] \prod_{v \in \mathrm{V}_{\text {out }}(\Gamma)}\left[\overline{\mathcal{H}}_{\mathrm{g}(v)}\left(\mu^{+}[v], I[v]-1\right)\right]\right]
\end{aligned}
$$

to obtain an equation in $A^{g-1}\left(\overline{\mathcal{M}}_{g, n}\right)$.

We study the precise contribution to the right side of (39) of the graphs $\Gamma_{i}$ characterized by (37) and (38). The graph $\Gamma_{i}$ has a unique possible twist ${ }^{22}$

$$
I(e)=m_{i}^{+} .
$$

${ }^{22}$ We use the condition $m_{i}^{+}>0$ here. 
The contribution of $\Gamma_{i}$ is

$$
m_{i}^{+} \cdot \epsilon_{*} \xi_{\Gamma_{i} *}\left[\left[\overline{\mathcal{H}}_{0}\left(m_{i}^{+},-1,-m_{i}^{+}-1\right)\right] \cdot\left[\overline{\mathcal{H}}_{g}\left(m_{1}^{+}, \ldots, m_{i-1}^{+}, m_{i}^{+}-1, m_{i+1}^{+}, \ldots, m_{n}^{+}\right)\right]\right]
$$

where we have

$$
\epsilon \circ \xi_{\Gamma_{i}}: \overline{\mathcal{M}}_{0,3} \times \overline{\mathcal{M}}_{g, n} \stackrel{\sim}{\longrightarrow} \overline{\mathcal{M}}_{g, n} .
$$

Using the isomorphism (40), the contribution of $\Gamma_{i}$ is simply

$$
\operatorname{Cont}\left(\Gamma_{i}\right)=m_{i}^{+} \cdot\left[\overline{\mathcal{H}}_{g}\left(m_{1}^{+}, \ldots, m_{i-1}^{+}, m_{i}^{+}-1, m_{i+1}^{+}, \ldots, m_{n}^{+}\right)\right] \in A^{g-1}\left(\overline{\mathcal{M}}_{g, n}\right) .
$$

The contribution of $\Gamma_{n}$ to the right side of (39) is special. Since by construction $m_{n}^{+}-1=m_{n}$, we see

$$
\operatorname{Cont}\left(\Gamma_{n}\right)=\left(m_{n}+1\right) \cdot\left[\overline{\mathcal{H}}_{g}\left(m_{1}, \ldots, m_{n}\right)\right]=\left(m_{n}+1\right) \cdot\left[\overline{\mathcal{H}}_{g}(\mu)\right] \in A^{g-1}\left(\overline{\mathcal{M}}_{g, n}\right)
$$

with $m_{n}+1 \neq 0$. The contributions of the graphs $\Gamma_{1}, \ldots, \Gamma_{n-1}$ are proportional to classes

$$
\left[\overline{\mathcal{H}}_{g}\left(\mu^{\prime}\right)\right] \in A^{g-1}\left(\overline{\mathcal{M}}_{g, n}\right)
$$

for nonnegative partitions $\mu^{\prime}$ with largest part larger than the largest part of $\mu$.

Lemma A2. Let $\mu=\left(m_{1}, \ldots, m_{n}\right)$ be holomorphic with no 0 parts. The class

$$
\left[\overline{\mathcal{H}}_{g}(\mu)\right] \in A^{g-1}\left(\overline{\mathcal{M}}_{g, n}\right)
$$

is determined via formula (39) by classes of the following four types:

(i) $\mathrm{H}_{g, \mu^{+}} \in A^{g}\left(\overline{\mathcal{M}}_{g, n}\right)$;

(ii) $\left[\overline{\mathcal{H}}_{g^{\prime}}\left(\mu^{\prime}\right)\right] \in A^{g^{\prime}}\left(\overline{\mathcal{M}}_{g^{\prime}, n^{\prime}}\right) \quad$ for $\quad\left(g^{\prime}, n^{\prime}\right) \stackrel{\circ}{<}(g, n) \quad$ and $\quad \mu^{\prime}=\left(m_{1}^{\prime}, \ldots, m_{n^{\prime}}^{\prime}\right) \quad$ strictly meromorphic;

(iii) $\left[\overline{\mathcal{H}}_{g^{\prime \prime}}\left(\mu^{\prime \prime}\right)\right] \in A^{g^{\prime \prime}-1}\left(\overline{\mathcal{M}}_{g^{\prime \prime}, n^{\prime \prime}}\right) \quad$ for $\quad\left(g^{\prime \prime}, n^{\prime \prime}\right) \stackrel{\circ}{<}(g, n) \quad$ and $\quad \mu^{\prime \prime}=\left(m_{1}^{\prime \prime}, \ldots, m_{n^{\prime \prime}}^{\prime \prime}\right)$ holomorphic;

(iv) $\left[\overline{\mathcal{H}}_{g}\left(\mu^{\prime \prime}\right)\right] \in A^{g-1}\left(\overline{\mathcal{M}}_{g, n}\right)$ for $\mu^{\prime \prime}=\left(m_{1}^{\prime \prime}, \ldots, m_{n}^{\prime \prime}\right)$ holomorphic with the largest part of $\mu^{\prime \prime}$ larger than the largest part of $\mu$.

Proof. The $\epsilon$ push-forward of the class (i) appears on the left of (39). Nontrivial simple star graphs not equal to $\Gamma_{1}, \ldots, \Gamma_{n}$ contribute (ii) via the center vertex and (iii) via the outlying vertices. The graphs $\Gamma_{1}, \ldots, \Gamma_{n-1}$ contribute (iv). We then solve (39) for the contribution $\left(m_{n}+1\right) \cdot\left[\overline{\mathcal{H}}_{g}(\mu)\right]$ of $\Gamma_{n}$.

A.6.3. Determination. We now combine Lemmas A1 and A2 to obtain the following basic result.

Theorem A3. Conjecture A effectively determines the classes

$$
\left[\overline{\mathcal{H}}_{g}(\mu)\right] \in A^{*}\left(\overline{\mathcal{M}}_{g, n}\right)
$$

both in the holomorphic and the strictly meromorphic cases. 
Proof. If $g=0$, the class $\left[\overline{\mathcal{H}}_{g}(\mu)\right]$ vanishes in the holomorphic case and is the identity $1 \in A^{*}\left(\overline{\mathcal{M}}_{0, n}\right)$ in the strictly meromorphic case. We proceed by induction with respect to the partial ordering $\stackrel{\circ}{<}$ on pairs defined by $(32)$.

If $\mu$ is strictly meromorphic, we apply Lemma A1. Conjecture A determines the class

$$
\mathrm{H}_{g, \mu} \in A^{g}\left(\overline{\mathcal{M}}_{g, n}\right) \text {. }
$$

The rest of the classes specified by Lemma A1 are strictly lower in the partial ordering $<$.

If $\mu$ is holomorphic and $\mu$ has parts equal to 0 , then either

$$
g=1, \quad \mu=(0, \ldots, 0), \quad \text { and }\left[\overline{\mathcal{H}}_{g}(0, \ldots, 0)\right]=1 \in A^{0}\left(\overline{\mathcal{M}}_{1, n}\right)
$$

or $\mu^{\prime}$ (obtained by removing the 0 parts) yields a class

$$
\left[\overline{\mathcal{H}}_{g}\left(\mu^{\prime}\right)\right] \in A^{g-1}\left(\overline{\mathcal{M}}_{g, n^{\prime}}\right)
$$

which is strictly lower in the partial ordering $\stackrel{\circ}{<}$. We then apply the pull-back (33) to determine $\left[\overline{\mathcal{H}}_{g}(\mu)\right] \in \overline{\mathcal{M}}_{g, n}$.

We may therefore assume $\mu$ is holomorphic with no 0 parts. We then apply Lemma A2. Conjecture A determines the class

$$
\epsilon_{*} \mathrm{H}_{g, \mu^{+}} \in A^{g-1}\left(\overline{\mathcal{M}}_{g, n}\right) .
$$

The rest of the classes specified by Lemma A2 either are strictly lower in the partial ordering $\stackrel{\circ}{<}$ or are:

(iv) $\left[\overline{\mathcal{H}}_{g}\left(\mu^{\prime \prime}\right)\right] \in A^{g-1}\left(\overline{\mathcal{M}}_{g, n}\right)$ for $\mu^{\prime \prime}=\left(m_{1}^{\prime \prime}, \ldots, m_{n}^{\prime \prime}\right)$ holomorphic with the largest part of $\mu^{\prime \prime}$ larger than the largest part of $\mu$.

To handle (iv), we apply descending induction on the largest part of $\mu$ in the holomorphic case. The base for the descending induction occurs when the largest part is $2 g-2$, then there are no partitions with larger largest part.

We have presented a calculation of the classes of the closures

$$
\overline{\mathcal{H}}_{g}(\mu) \subset \overline{\mathcal{M}}_{g, n}
$$

of the moduli spaces of canonical divisors on nonsingular curves via Conjecture $\mathrm{A}$ and the virtual components of the moduli space of twisted canonical divisors. Theorem A3 yields an effective method with result

$$
\left[\overline{\mathcal{H}}_{g}(\mu)\right] \in R^{*}\left(\overline{\mathcal{M}}_{g, n}\right) .
$$

The $g=2$ cases with $\mu=(3,-1)$ and $\mu=(2,1,-1)$ discussed in $\S$ A.5 may also be viewed as steps in the recursion of Theorem A3 to calculate

$$
\overline{\mathcal{H}}_{2,(2)} \in R^{1}\left(\overline{\mathcal{M}}_{2,1}\right) \quad \text { and } \quad \overline{\mathcal{H}}_{2,(1,1)} \in R^{1}\left(\overline{\mathcal{M}}_{2,2}\right) \text {, }
$$

respectively. Since we already have formulas for the classes (41), the calculations serve to check Conjecture A. 


\section{References}

1. M. Bainbridge, D. Chen, Q. Gendron, S. Grushevsky and M. Möller, in preparation.

2. P. Belorousski and R. Pandharipande, A descendent relation in genus 2, Ann. Sc. Norm. Super. Pisa Cl. Sci. 29 (2000), 171-191.

3. L. Caporaso and E. Sernesi, Recovering plane curves from their bitangents, J. Algebraic Geom. 12 (2003), 225-244.

4. D. Chen, Degenerations of abelian differentials, preprint, 2015, arXiv:1504.01983.

5. D. Chen And N. TARASCA, Loci of curves with subcanonical points in low genus, preprint, arXiv:1501.02235.

6. E. Clader and F. JANDA, Pixton's double ramification cycle relations, preprint, arXiv: 1601.02871.

7. M. Cornalba, Moduli of curves and theta-characteristics, in Lectures on Riemann surfaces (Trieste, 1987), pp. 560-589 (World Scientific Publications, 1989).

8. D. Eisenbud And J. Harris, The Kodaira dimension of the moduli space of curves of genus $\geqslant 23$, Invent. Math. 90 (1987), 359-387.

9. A. Eskin, H. MAsur AND A. Zorich, Moduli spaces of abelian differentials: the principal boundary, counting problems and the Siegel-Veech constants, Publ. Math. Inst. Hautes Études Sci. 97 (2003), 61-179.

10. A. Eskin And A. OKounkov, Asymptotics of the numbers of branched coverings of a torus and volumes of moduli spaces of holomorphic differential, Invent. Math. 145 (2001), 59-103.

11. C. Faber And R. PAndharipande, Relative maps and tautological classes, J. Eur. Math. Soc (JEMS) 7 (2005), 13-49.

12. G. FARKAs, The birational type of the moduli space of even spin curves, Adv. Math. 223 (2010), 433-443.

13. W. Fulton, Intersection Theory (Springer, Berlin, 1984).

14. Q. Gendron, The Deligne-Mumford and the incidence variety compactifications of the strata of $\Omega \mathcal{M}_{g}$, preprint, arXiv:1503.03338.

15. T. Graber and R. Pandharipande, Constructions of nontautological classes on moduli spaces of curves, Michigan Math. J. 51 (2003), 93-109.

16. R. HAIN, Normal Functions and the Geometry of Moduli Spaces of Curves (ed. G. FARKAS AND I. Morrison), Handbook of Moduli, Volume I, pp. 527-578 (International Press, 2013).

17. H. Hilton, Plane Algebraic Curves (Clarendon, Oxford, 1920).

18. F. Janda, R. Pandharipande, A. Pixton and D. Zvonkine, Double ramification cycles on the moduli spaces of curves, preprint, arXiv:1602.04705.

19. F. JANDA, in preparation.

20. Y.-H. Kiem And J. Li, Localizing virtual classes by cosections, J. Amer. Math. Soc. 26 (2013), 1025-1050.

21. M. Kontsevich And A. Zorich, Connected components of the moduli spaces of abelian differentials with prescribed singularities, Invent. Math. 153 (2003), 631-678.

22. H. MASUR, Interval exchange transformations and measured foliations, Ann. of Math. (2) 115 (1982), 169-200.

23. R. Pandharipande, A. Pixton and D. Zvonkine, Relations on $\overline{\mathcal{M}}_{g, n}$ via 3-spin structures, J. Amer. Math. Soc. 28 (2015), 279-309.

24. A. Pixton, Double ramification cycles and tautological relations on $\overline{\mathcal{M}}_{g, n}$, preprint, 2014. 
25. A. Polishchuk, Moduli spaces of curves with effective $r$-spin structures, in Gromov-Witten Theory of Spin Curves and Orbifolds, Contemporary Mathematics, Volume 403, p. 20 (American Mathematical Society, Providence, RI, 2006).

26. A. Sauvaget and D. Zvonkine, in preparation.

27. W. A. VeEch, Moduli spaces of quadratic differentials, J. Anal. Math. 55 (1990), 117-171.

28. S. Wolpert, Infinitesimal deformations of nodal stable curves, Adv. Math. 244 (2013), 413-440. 